\title{
Intestinal GCN2 controls Drosophila systemic growth in response to Lactiplantibacillus plantarum symbiotic cues encoded by r/tRNA operons
}

\author{
Grenier T. ${ }^{1}$, Consuegra J. ${ }^{1}$, Galvao Ferrarini M. ${ }^{2,3}$, Matos RC. ${ }^{1}$, Akherraz H. ${ }^{1}$, Gillet \\ B. ${ }^{1}$, Hughes S. ${ }^{1}$, Leulier $\mathrm{F}^{1^{*}}$.
}

1. Institut de Génomique Fonctionnelle de Lyon (IGFL), Ecole Normale Supérieure de Lyon, CNRS UMR 5242, Université Claude Bernard Lyon 1, 69364 Lyon Cedex 07, France

2. Univ Lyon, INSA Lyon, INRAE, BF2I, UMR 203, 69621 Villeurbanne, France

10 3. Laboratoire de Biométrie et Biologie Évolutive, UMR 5558, Université Lyon 1, Université Lyon, Villeurbanne, France

*corresponding author: francois.leulier@ens-lyon.fr

\section{Abstract}

Symbiotic bacteria interact with their host through the release of symbiotic cues. Here, we took advantage of the mutualism between Drosophila and Lactiplantibacillus plantarum (Lp) to investigate a novel mechanism of host-symbiont interaction. Using chemically-defined holidic diets, we found that association with Lp improves the growth of larvae fed on amino acid-imbalanced diets, even though Lp cannot produce the limiting amino acid. We show that in this context Lp supports its host's growth through a molecular dialog that requires functional operons encoding ribosomal and transfer RNAs ( $r$ /tRNAs) in Lp and the GCN2 kinase in Drosophila's enterocytes. Our data indicate that products of Lp r/tRNAs loci activate GCN2 in a subset of larval enterocytes, a mechanism necessary to remodel the intestinal transcriptome and ultimately to support anabolic growth. Our findings unravel a novel beneficial molecular dialog between host and microbes, which relies on a non-canonical role of GCN2 as a mediator of non-nutritional symbiotic cues encoded by r/tRNA operons.

\section{Introduction}

Animals have evolved and live in symbiosis with a great diversity of microbes. Symbiotic microbes strongly influence various aspects of their host's physiology, 35 metabolism and behaviour. The fruit fly Drosophila melanogaster (hereinafter referred to as Drosophila) is a powerful model to study the mechanisms underlying the interactions between host and symbiotic microbes. Indeed, Drosophila harbours simple bacterial communities, which individual components can be cultured aerobically. Moreover, Drosophila can easily be bred axenically, allowing large-scale gnotobiotic studies. Finally, Drosophila's main bacterial symbionts can be genetically engineered, enabling deep mechanistical studies on both the host side and the microbe side. In the past decade, Drosophila's symbiotic microbes were shown to modulate their host's post-embryonic growth (Shin et al., 2011; Storelli et al., 2011), reproduction (Elgart et al., 2016; Gould et al., 2018), lifespan (Keebaugh et al., 2019; Yamada et al., 2015), metabolism (Gnainsky et al., 2021; Kamareddine et al., 2018; 
Newell and Douglas, 2014), immunity (latsenko et al., 2018), social behaviour (Chen et al., 2019; Sharon et al., 2010) and food preference (Kim et al., 2021; LeitãoGonçalves et al., 2017). The mechanisms may rely on symbiotic cues: molecules produced by symbiotic microbes that interact with the host's signalling pathways. Symbiotic cues can be nutrients: for instance, amino acids (AA) produced by symbiotic bacteria can inhibit the production of the neuropeptide CNMamide in the gut, which represses preference for amino acids (Kim et al., 2021). In addition, symbiotic cues can be non-nutritional. Sensing of symbiotic bacteria's cell wall components by gut cells leads to the production of digestive enzymes, which helps the larva digest the dietary polypeptides and improves its growth (Erkosar et al., 2015; Matos et al., 2017). Acetate produced by symbiotic microbes alters the epigenome of enteroendocrine cells, which stimulates the secretion of the hormone Tachykinin (Tk) (Jugder et al., 2021). Tk then promotes lipid utilization in nearby enterocytes (Kamareddine et al., 2018). Beyond these examples, we posit that symbiotic cues may be widespread in Drosophila-microbes symbiosis but their nature remains elusive. Hence, identifying them is an important goal of the field of host-symbionts interactions (Selosse et al., 2014).

In this study, we sought to identify new non-nutritional symbiotic cues that allow

Drosophila's symbiotic bacteria to influence the physiology of their host. As a readout, we used Drosophila's post-embryonic growth. Drosophila's growth phase (larval stages) depends widely on the nutritional environment: larvae reach metamorphosis (pupariation) in 4-5 days in optimal nutritional conditions or up to 15-20 days in severe malnutrition conditions (Erkosar et al., 2013; Tennessen and Thummel, 2011). Nutrition regulates larval growth through the action of nutrient-sensing regulatory pathways, especially amino acids (AA)-sensing pathways. The main AA (or lack of-) sensing pathways are the Target-of-Rapamycin (TOR) kinase pathway and the general control nonderepressible 2 (GCN2) kinase (Gallinetti et al., 2013). Both kinases were first described in yeast (Dever et al., 1992; Heitman et al., 1991) and orthologous pathways were found in virtually all eukaryotes, including Drosophila (Olsen et al., 1998; Zhang et al., 2002). The TOR kinase forms two protein complexes: mTORC1 and mTORC2, which can be activated by many cues (Laplante and Sabatini, 2009). Especially, mTORC1 responds to high intracellular AA levels through the action of AA transporters and AA-binding cytosolic proteins; conversely, AA scarcity 80 represses TOR activity (Goberdhan et al., 2016). Once activated, TOR increases translation through phosphorylation of 4E-BP and S6K (Ma and Blenis, 2009) and promotes systemic growth of Drosophila larvae (Colombani et al., 2003). GCN2 is activated by several cues (Donnelly et al., 2013). The best-characterized cue is the quantity of uncharged tRNAs, a signature of hampered protein synthesis reflecting a 85 scarcity in intracellular AA (Masson, 2019). Activation of GCN2 causes a global translational repression through phosphorylation of the Eukaryotic Initiation Factor 2 $($ elF2 $\alpha)$ (Teske et al., 2011) except for a subset of mRNAs which translation is increased. Among these mRNAs, transcription factors such as ATF4 then promote adaptation to stress (Harding et al., 2003). In addition to its cell-autonomous effects, 
90 the GCN2 pathway has systemic effects in Drosophila. Ubiquitous knock-down of GCN2 in Drosophila larvae causes developmental delay (Malzer et al., 2013). Moreover, GCN2 is involved in the regulation of other physiological processes. Activation of GCN2 in dopaminergic neurons of the larval brain (Bjordal et al., 2014) or in the enterocytes of adult flies (Kim et al., 2021) trigger a marked behavioural response leading to the avoidance of diets with an imbalanced AA composition. In addition, GCN2 in adult enterocytes regulates gut plasticity in response to AA/sugar imbalance (Bonfini et al., 2021). Furthermore, GCN2 in the midgut or in the fat body is necessary for lifespan extension under dietary restriction (Kim et al., 2020). Finally, GCN2 in the fat body represses reproduction under AA scarcity (Armstrong et al., 2014).

Although undernutrition greatly delays the development of Germ-Free (GF) Drosophila larvae (i.e. larvae lacking a microbiota), such delay can be buffered by the association of GF larvae with certain strains of symbiotic microbes (Gould et al., 2018; Keebaugh et al., 2018; Shin et al., 2011; Storelli et al., 2011). Growth promotion relies partly on nutrient provision: for instance, the symbiotic bacterium Lactiplantibacillus plantarum (Lp) can provide certain AA, which allows the larva to grow in the absence of these specific AA (Consuegra et al., 2020a). We sought to identify mechanisms of growth promotion that do not rely on AA provision. Therefore, we used a holidic diet (HD, diet 110 composed of purified nutrients) (Piper et al., 2017) to create AA imbalance by specifically decreasing the quantity of an AA that Lp cannot synthesize. Interestingly, we found that Lp can still promote larval growth in these conditions. The mechanism thus does not rely on AA provision, nor on stimulation of intestinal proteases (Erkosar et al., 2015) because the HD contains only free AA. Instead, we found that it depends

115 on the production of ribosomal and transfer RNA by Lp, which leads to the activation of GCN2 in enterocytes. GCN2 activation results in a remodelling of the gut transcriptome towards epithelium maturation and altered metabolic activity, which may support systemic growth. Our study suggests that on top of its canonical role as a cellular sensor of uncharged eukaryotic tRNA, GCN2 is also a mediator of nonnutritional symbiotic cues encoded by bacterial r/tRNA operons.

\section{Results}

\section{Association with Lp rescues amino acid imbalance}

We used a HD which AA composition is based on Drosophila's exome (FLY AA diet, (Piper et al., 2017). This diet is well-balanced in AA and allows optimal reproduction, lifespan and growth. AA imbalance can be generated by decreasing the concentration of any essential AA (EAA), which then becomes limiting for animal fitness. Imbalanced

130 diets cause defects in Drosophila's growth, reproduction and lifespan (Piper et al., 2017). In a previous study, we used HD to identify which AA can be synthesized by Lp and provided to the Drosophila larva. Lp cannot rescue the development of larvae on a diet that completely lacks an EAA that it cannot synthetize such as Isoleucine, 
Leucine and Valine (Consuegra et al., 2020a). Here, we wondered whether or not Lp could rescue the effects of AA imbalance due to limitations in EAA. To this end, we selectively decreased the concentration of each EAA by $70 \%$ from the FLY AA diet and measured the time of development of larvae (expressed as D50, i.e. the median time of entry into metamorphosis). As expected, decreasing the concentration of any EAA causes growth delay in GF larvae. Decreasing the amount of certain EAA (Ile,

140 Lys, Thr, Trp, Val) completely blocks the development of GF larvae, which stall at the L2 or L3 stage. Decreasing the amount of the other EAA (Arg, His, Met, Leu, Phe) causes an important developmental delay in GF larvae compared to the FLY AA diet (Fig. 1A). In order to compare the different conditions, we aimed at finding a setup in which GF larvae are able to reach pupariation, with a strong developmental delay. We

145 thus imposed a less severe AA imbalance by only decreasing the concentration of each $\mathrm{EAA}$ by $60 \%$. We observed a similar trend: decreasing the amount of lle, Lys, Thr, Trp or Val yields an important developmental delay in GF larvae, whereas decreasing the amount of Arg, His, Met, Leu or Phe results in a minor developmental delay (Fig. 1B). This confirms that some EAA seem to be more important than others

150 for the development of GF larvae; alternatively, it is possible that the FLY AA diet does not contain enough of these EAA (Ile, Lys, Thr, Trp, Val) and too much of the others (Arg, His, Met, Leu, Phe).

Association with $L p$ rescues the effects of limitation in any EAA (Fig. 1A,B). Lp's genome does not encode the enzymes necessary for the synthesis of BranchedChained AA (BCAA: Leucine, Isoleucine and Valine) (Martino et al., 2016; Saguir and de Nadra, 2007; Teusink et al., 2005), and thus Lp cannot provide them to Drosophila (Consuegra et al., 2020a; Kim et al., 2021). Therefore, we were interested by the fact that $L p$ rescues the developmental delay caused by BCAA limitation, which it cannot

160 synthetize de novo. We thus decided to focus on diets limited in Valine (Val) to decipher the mechanisms underlying such beneficial effect of $L p$ on the growth of its host. We thereafter refer to the FLY AA diet as "balanced diet", and to the FLY AA -60\% Val diet as "imbalanced diet". Decreasing Val by $60 \%$ results in a strong delay in the growth of GF larvae, which is almost completely rescued by association with Lp (Fig. 1C).

165 Replacing the missing Val with an equal quantity of another EAA (Leu or His) does not improve the development of GF larvae (Fig. 1D). This shows that the delay observed on imbalanced diet is due to AA imbalance rather than total AA scarcity. Of note, further decreasing Val concentration $(-80 \%,-90 \%)$ is lethal to GF larvae, but not to Lpassociated larvae (Fig. S1A). Completely removing Val from the diet is lethal to both

170 GF larvae and Lp-associated larvae as $\mathrm{Lp}$ is a Val auxotroph (Consuegra et al., 2020a). On the contrary, increasing Val by $100 \%$ compared to its initial levels does not impact the development of GF or Lp-associated larvae (Fig. S1B). Moreover, egg-topupa survival is not impacted by AA imbalance nor by association with Lp (Fig. S1C). Finally, supplementing the GF larvae with Heat-Killed (HK) Lp does not rescue the 175 effects of an imbalanced diet on larval growth, which shows that the Val brought by the inoculation of $\mathrm{Lp}$ at the beginning of the experiment is not sufficient to restore Val levels required for larval growth (Fig. S1D). Taken together, these results demonstrate that 
Lp can rescue the effects of AA imbalance due to a single dietary EAA limitation on larval growth through a mechanism independent of AA providing. Instead, we posit that Lp promotes physiological adaptation of its host to AA imbalance to support larval growth.

Figure 1
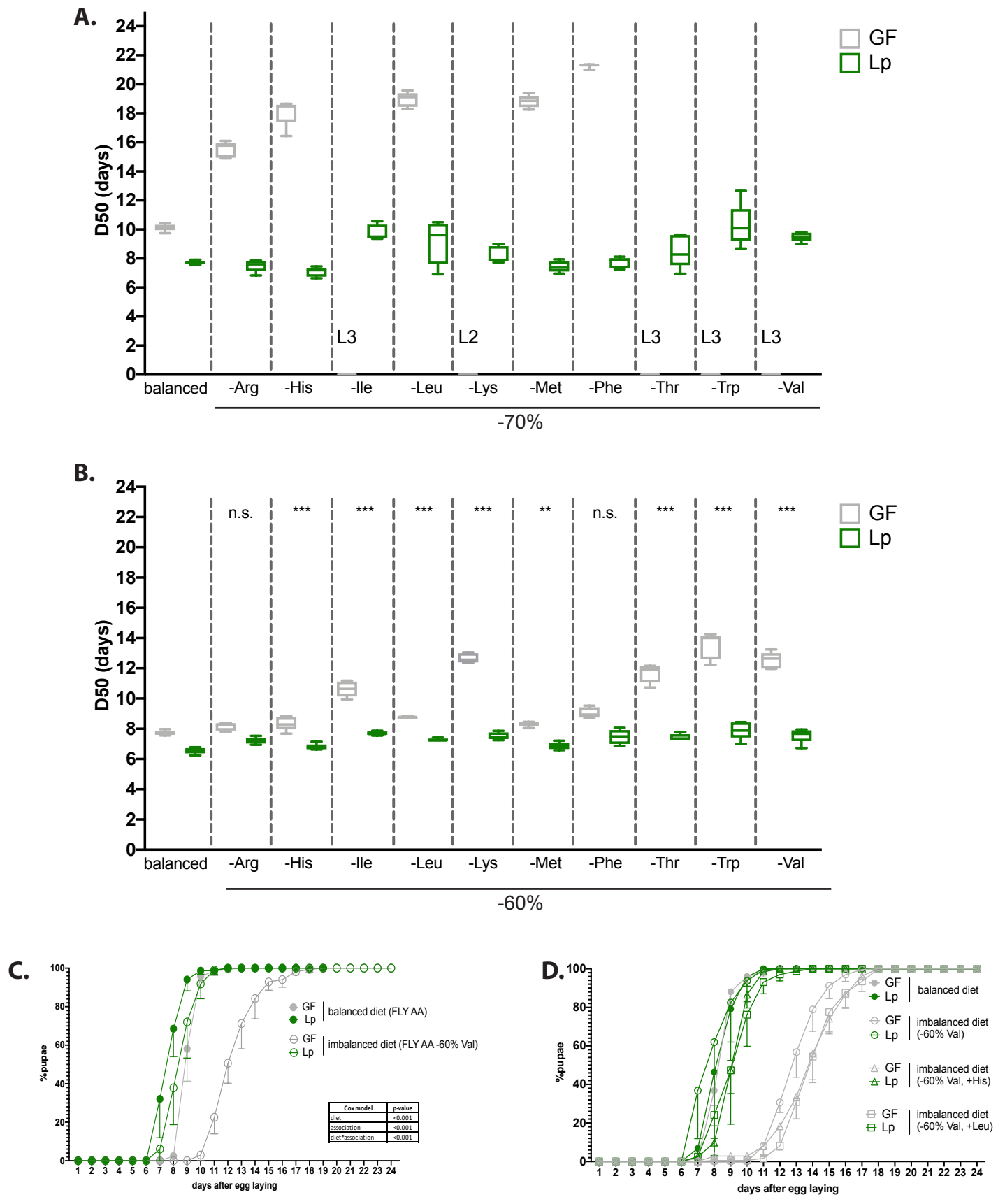

Fig. 1 Lp rescues the developmental delay due to AA imbalance.

185 (A,B) Developmental timing of GF larvae (grey) and Lp-associated larvae (green) on FLY AA diets with a $-70 \%(A)$ or $-60 \%$ (B) decrease in each EAA. Boxplots show maximum, minimum and median D50 (median time of pupariation) of each replicate. L2: larvae stalled at the L2 stage. L3: larvae stalled at the L3 stage. For each diet in (B), we used a Cox proportional hazards model to test the effect of the diet, association with Lp, and the interaction between these two parameters. We show the p-values of 
190 the interactions between diet and association with Lp after correction by the FDR method. n.s.: nonsignificant, ${ }^{* *}$ : $p$-value $<0.01,{ }^{* * *}$ : $p$-value $<0.001$. (C) Developmental timing of larvae raised on balanced diet (FLY AA, filled circles) or imbalanced diet (FLY AA-60\% Val, empty circles). The graph represents the total fraction of emerged pupae over time as a percentage of the final number of pupae. We used a Cox proportional hazards model to test the effect of the diet, the association with $L p$, and the interaction between these two parameters (D) Developmental timing of larvae raised on balanced diet (FLY AA, filled circles), on imbalanced diet (FLY AA Val-60\%, empty circles), on imbalanced diet adjusted with His (FLY AA Val $-60 \%+$ His, triangles) or on imbalanced diet adjusted with Leu (FLY AA Val $-60 \%+$ Leu, squares). The graph represents the total fraction of emerged pupae over time as a percentage of the final number of pupae.

\section{Figure S1}

A.

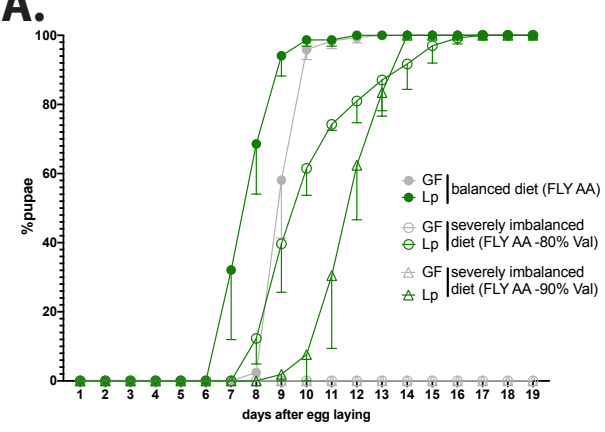

C.

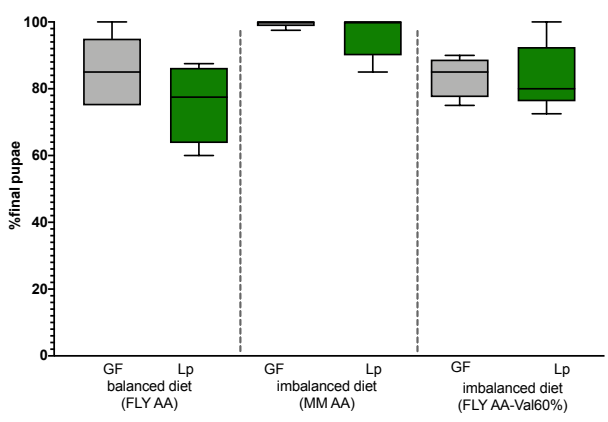

B.

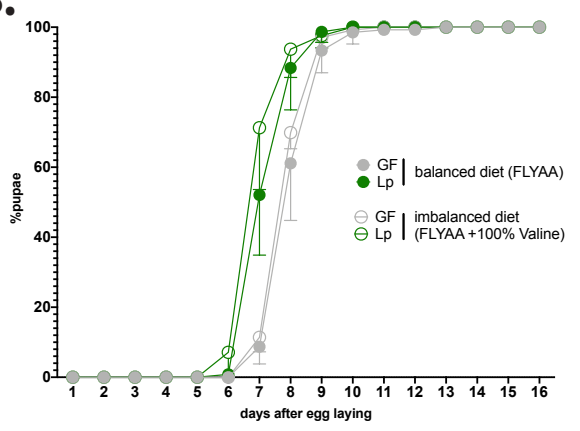

D.

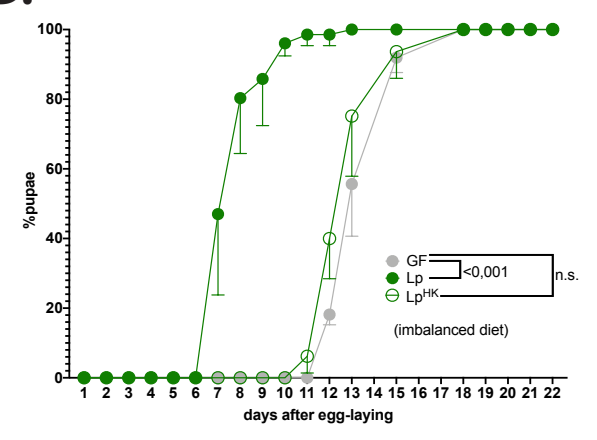

Fig. $\mathbf{S 1}$

(A) Developmental timing of larvae raised on balanced diet (FLY AA, filled circles) or on severely imbalanced diets (FLY AA - $80 \%$ Val, empty circles, FLY AA - $90 \%$ Val, empty triangles) in GF condition (grey) or Lp-associated conditions (green). The graph represents the total fraction of emerged pupae over time as a percentage of the final number of pupae. GF larvae reared on severely imbalanced diets ( $-80 \%$ or $-90 \%$ Val) did not reach pupariation. (B) Developmental timing of larvae raised on balanced diet (FLY AA, filled circles) or on imbalanced diet due to excess Val (FLY AA $+60 \%$ Val, empty circles). (C) Egg-to-pupa survival of GF larvae (in grey) and Lp-associated larvae (green) on balanced diet and imbalanced diets. Survival was calculated as the final number of pupae divided by the initial number of eggs (i.e. 40 per replicate). We performed a Kruskal-Wallis test followed by post-hoc Dunn's tests to compare each condition to the condition GF on balanced diet and found no statistically significant difference. (D) Developmental timing of larvae raised on imbalanced diet (FLY AA Val -60\%) in GF conditions (grey), in Lp-associated conditions (filled green circles) or after supplementation with the same quantity of Heat-Killed (HK) Lp (empty green circles). We used a Cox proportional hazards model to compare the effect of $L p$ and HK Lp to the GF condition. 


\section{Rescue of AA imbalance by Lp requires functional r/tRNAs operons in Lp}

In order to decipher how $L p$ rescues the effects of AA imbalance on host growth, we performed a genetic screen using a transposon insertion library of Lp (Fig. 2A). This library is composed of 2091 mutants, each carrying a transposon randomly inserted in the chromosome, including 1218 insertions inside open reading frames (Matos et al., 2017). We mono-associated GF larvae with each mutant of the library and looked for transposon insertions in Lp's genome altering the capacity of $L p$ to support larval development on a severely imbalanced diet (FLY AA -80\%Val, Fig. S1A). For each mutant, we calculated the D50 (median time of entry into metamorphosis) and normalized it into a z-score. We applied a threshold of z-score $>2.5$ and identified 32 insertional mutants. Association with these mutants thus results in a delayed time of larval development on a severely imbalanced diet (Fig. 2B). To validate these 32 candidates, we individually re-tested them in multiple (5) replicates. We compared the development of larvae associated with the 32 candidates to larvae associated with an intergenic region insertion strain showing a WT-like phenotype (mutant B02.04, zscore $=0.65$ ). Thus, we discarded 23 false positives and retained only 9 candidates which result in a significant and robust developmental delay on an imbalanced diet upon association (Fig. 2C).

240 Upon association with Drosophila larvae Lp grows on the fly food and constantly and repetitively transits through the larval gut (Storelli et al., 2018). The quantity of live bacteria present in the food can greatly impact the growth-promoting capacity of Lp (Consuegra et al., 2020b; Keebaugh et al., 2018). As a consequence, a Lp strain that grows poorly on the food matrix would not support larval growth. Since we wanted to exclude such candidates, we tested the growth of the 9 candidates on imbalanced HD, in the presence of larvae (Fig. 2D). 3 candidates (B08.06, F09.11 and H04.06) show growth defects on the diet and thus were not retained for further analysis. On the contrary, the remaining 6 candidates (B12.11, C08.20, C09.09, D12.09, D12.16, F07.08) show no growth defect. Moreover, they do not show any impairment at colonizing the larval gut (Fig. 2E). For further characterization, we tested whether the effect of the mutations may also be observed on a moderately imbalanced diet $(-60 \%$ Val). On such diet, the mutations B12.11 and C08.20 do not significantly impact Lp's ability to rescue the developmental delays of larvae. On the contrary, larvae associated with the mutants C09.09, D12.09, D12.16 and F07.08 are still delayed compared to the larvae associated with the WT-like mutant B02.04 (Fig. 2F), though the difference is less important than on a severely imbalanced diet (-80\% Val, Fig. $2 \mathrm{C}$ ).

Next, we sequenced the genomes of the 6 selected candidates to determine in which genomic regions the transposons were inserted. Interestingly, 4 out of the 6 candidates (mutants C09.09, D12.09, D12.16 and F07.08) showed independent transposon

260 insertions in operons containing genes encoding transfer RNAs (tRNAs) and/or ribosomal RNAs (rRNA) (Fig. 2G). These four mutants are also the ones that seem to most affect larval development on imbalanced diet (Fig. 2F). The genome of $L p$ 
contains 5 operons encoding r/tRNAs. C09.09 and F07.08 display independent insertions in the same operon, but at different loci (upstream the 16S rRNA for C09.09 and inside the 23S rRNA for F07.08, Fig. 2G). We extracted total RNA from Lp cultured in liquid HD and found that despite the redundancy of r/tRNAs operon in Lp's genome the F07.08 insertion mutant displays a $\sim 3$ to 4 -fold reduction of $16 \mathrm{~S}$ (Fig. S2A) and $23 \mathrm{~S}$ (Fig. S2B) rRNA compared to B02.04, indicating that altering the sequence of a single r/tRNA operon is sufficient to compromise global rRNA levels in Lp cells.

To confirm the importance of $r$ /tRNA operons in supporting larval growth, we generated a deletion mutant of the whole operon identified in the insertion mutants C09.09 and F07.08 $\left(\Delta \mathrm{op}_{\mathrm{r} / \mathrm{RNA}}\right)$ by homology-based recombination (Matos et al., 2017). $\Delta \mathrm{op}_{\mathrm{r} / \mathrm{RNA}}$ shows the same phenotype as the insertion mutants in terms of lack of rescue of AA imbalance (Fig. S2C), growth on imbalanced HD in the presence of larvae (Fig. S2D), larval gut colonization (Fig. S2E) and reduction of the expression of the 16S (FigS2F) and $23 S$ (Fig. S2G) rRNA ( 3-fold reduction). The fact that the chromosome of Lp contains five operons encoding $r / t R N A s$ may explain why deleting one of such operons does not have a major impact on bacterial fitness. However, it seems that the production of r/tRNAs (or any other yet unknown product of this loci) from these operons is extremely rate limiting to support Drosophila growth upon AA imbalance. 


\section{Figure 2}

A.

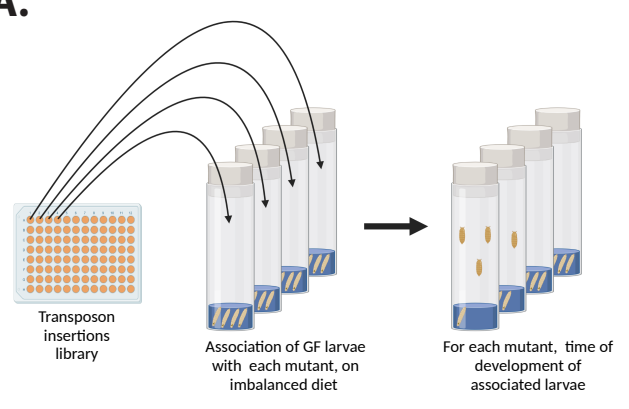

C.
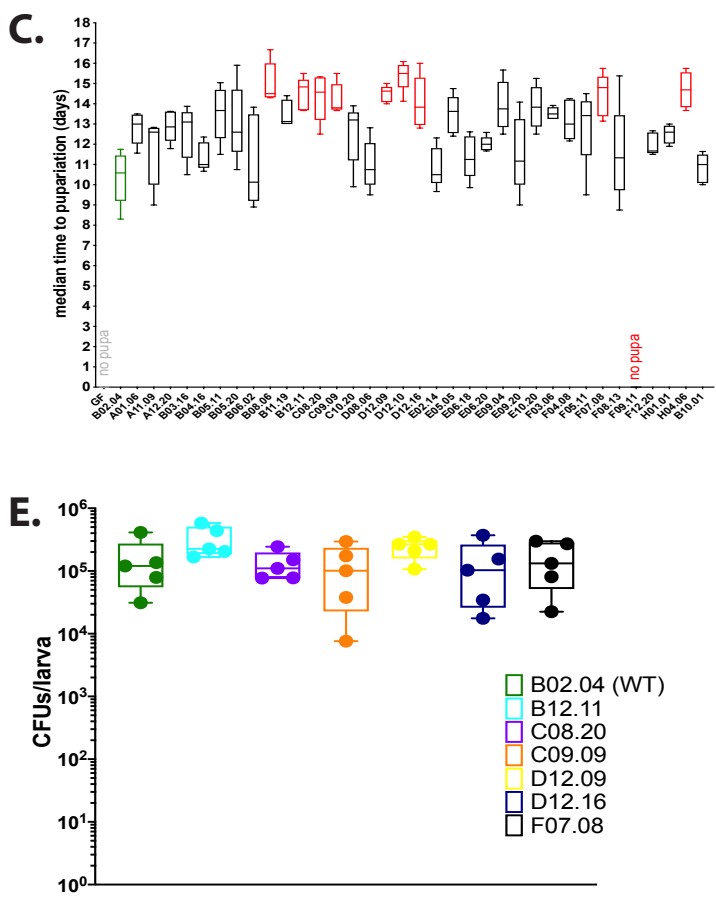

B.

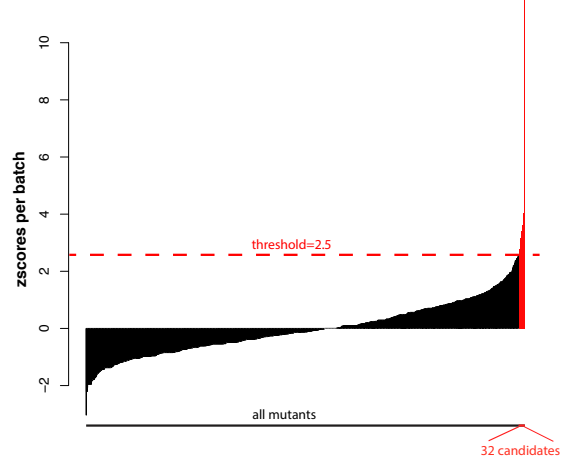

D.

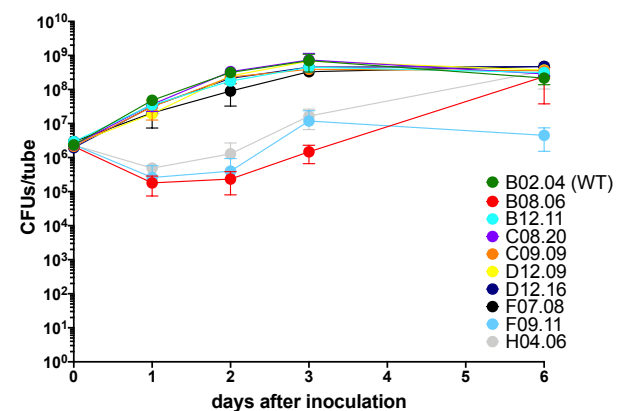

F.

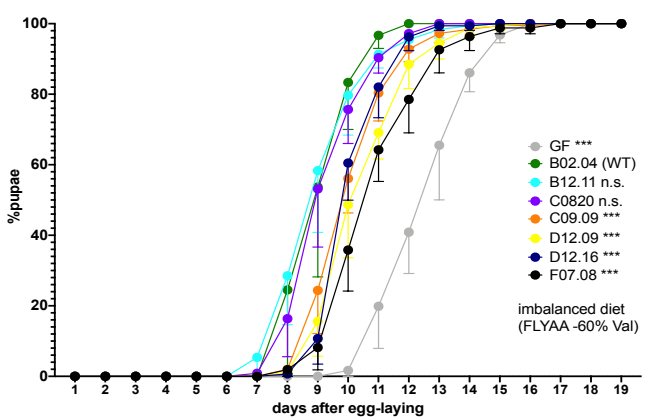

G.
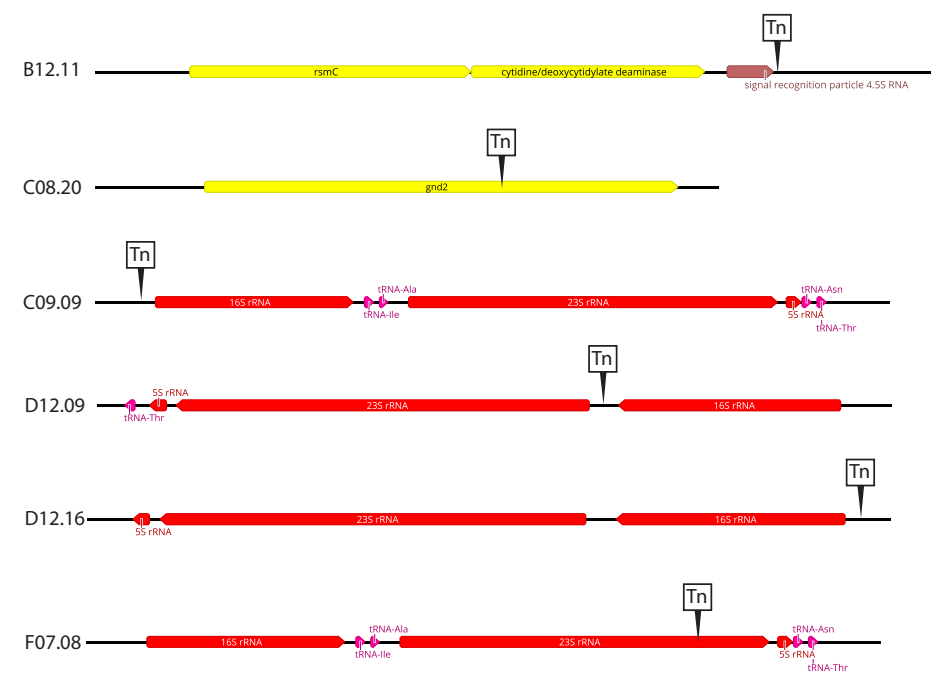

Fig. 2 The operons encoding ribosomal and transfer RNAs in Lp are necessary for Lp to rescue the delay due to AA imbalance.

(A) Representation of the genetic screen. (B) Result of the screen: for each mutant (X-axis), we calculated the median time of development of associated larvae and normalized it into a $\mathrm{Z}$-score $(\mathrm{Y}$ - 
bioRxiv preprint doi: https://doi.org/10.1101/2021.10.31.466661; this version posted December 21, 2021. The copyright holder for this preprint (which was not certified by peer review) is the author/funder, who has granted bioRxiv a license to display the preprint in perpetuity. It is made available under aCC-BY-NC 4.0 International license.

axis). We selected the 32 candidates that yielded a z-score>2.5. (C) Developmental timing of GF larvae (grey) and larvae associated with WT-like mutant B02.04 (green) or the 32 candidate mutants from the genetic screen, on a severely imbalanced diet (FLY AA $-80 \%$ Val). GF larvae and larvae associated with mutant F09.11 did not reach pupariation. Boxplots show maximum, minimum and median of D50 (median time of pupariation) of each replicate. We performed a Kruskal-Wallis test followed by post-hoc Dunn's tests to compare all mutants to B02.04. In red: statistically significant difference with B02.04 (pvalue $<0.05$ ). (D) Growth of the 9 candidates on imbalanced diet (FLY AA -60\% Val), in association with larvae. The graph shows the quantity of Colony-Forming Units (CFUs) of Lp over time. (E) Colonization of the larval gut by the 6 remaining candidates, on imbalanced diet (FLY AA -60\% Val). The graph shows the quantity of Colony-Forming Units (CFUs) of Lp per larva. We performed a Kruskal-Wallis test followed by post-hoc Dunn's tests to compare each candidate to B02.04 and found no statistically significant difference. (F) Developmental timing of larvae raised on imbalanced diet (FLY AA-60\% Val), in GF condition or in association with each one of the six candidates or with B02.04. The graph represents the total fraction of emerged pupae over time as a percentage of the final number of pupae. We used a Cox proportional hazards model to compare the effect of each candidate to the effect of B02.04. The p-values were adjusted by the Tukey method. n.s.: non-significant. ${ }^{* *}$ : $p$-value $<0.001$. (G) Representation of the six transposon insertions. Tn: transposon. rspC: 16S rRNA methyltransferase. gnd2: phosphogluconate dehydrogenase. Of note, C09.09 and F07.08 show two independent insertions in the same r/tRNA operon. 


\section{Fig. 52}

A.

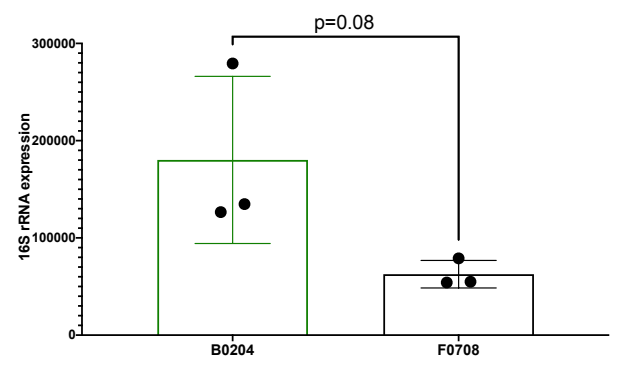

C.

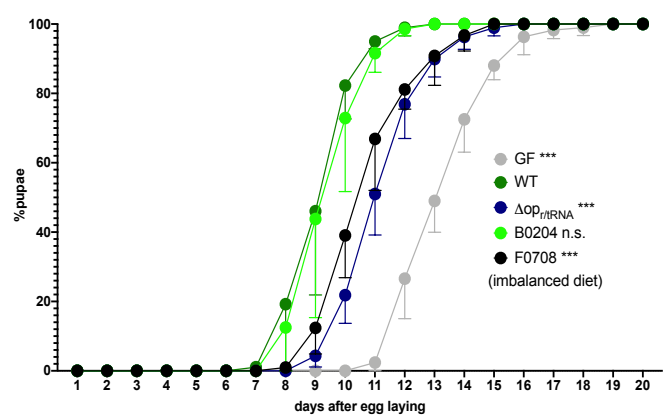

D.

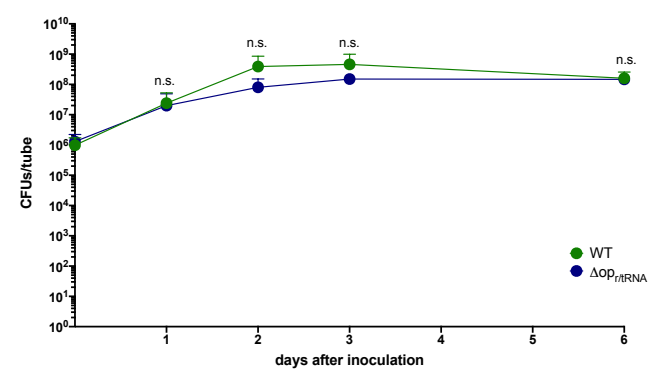

F.

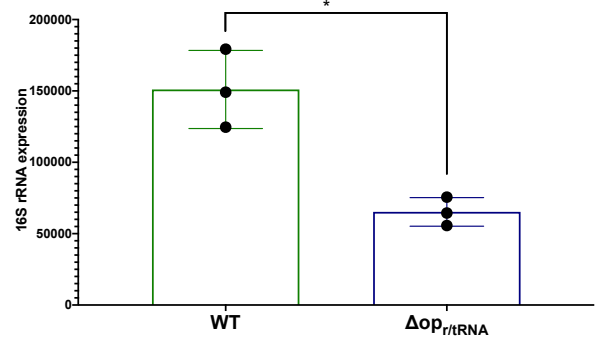

B.

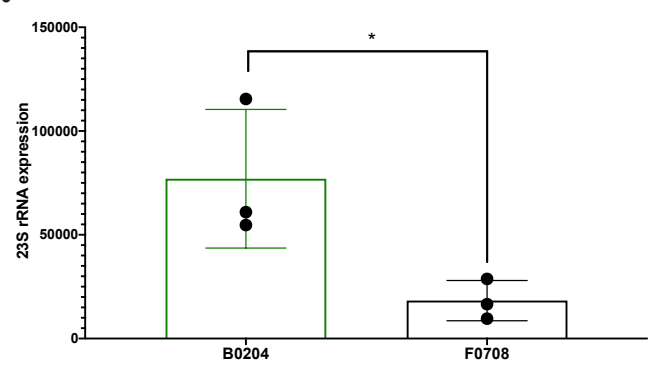

E.

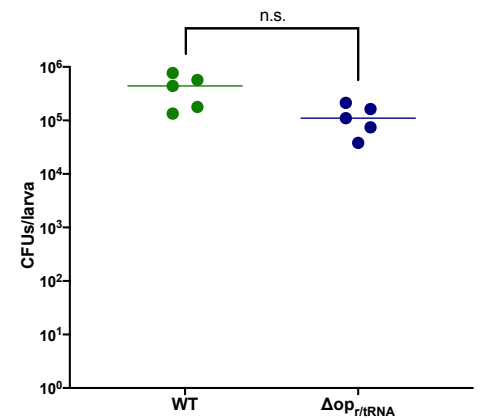

G.

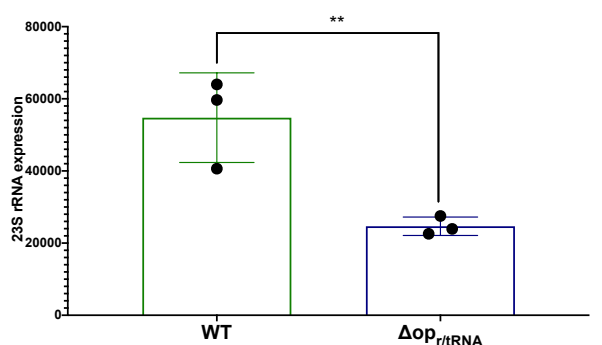

Fig. S2

(A,B) Expression of 16S (A) and 23S (B) rRNA of WT-like mutant B02.04 and r/tRNA insertion mutant F07.08. Expression is normalized with the expression of gyrB using the formula $2^{\mathrm{Ca}(g y r B)-C q(R N A)}$. We performed a Student's t-test to compare the two conditions. *: p-value $<0.05$. (C) Developmental timing of larvae raised on imbalanced diet (FLY AA-60\% Val), in GF condition or in association with Lp (dark green), WT-like mutant B02.04 (light green), Lp $\triangle \mathrm{Op}$ rtiRNA (dark blue) or r/tRNA insertion mutant F07.08 (black). The graph represents the total fraction of emerged pupae over time as a percentage of the final number of pupae. We used a Cox proportional hazards model to compare the effect of each candidate to the effect of $L p$. The p-values were adjusted by the Tukey method. n.s.: non-significant. ${ }^{* * *}$ : $p$ value $<0.001$. (D) Growth of $L p$ (dark green) and $L p \triangle \mathrm{Op}_{\text {rtrknA }}$ (dark blue) on imbalanced diet (FLY AA $60 \%$ Val), in association with larvae. The graph shows the quantity of Colony-Forming Units (CFUs) of Lp over time. We performed a Mann-Whitney test at each time point and found no statistically significant 
difference. (E) Colonization of the larva gut by $L p$ (dark green) and Lp $\triangle \mathrm{op}_{\mathrm{r} / \mathrm{tRNA}}$ (dark blue) on imbalanced diet (FLY AA -60\% Val). The graph shows the quantity of Colony-Forming Units (CFUs) of Lp per larva. We performed a Mann-Whitney test and found no statistically significant difference. $(\mathbf{F}, \mathbf{G})$ Expression of $16 \mathrm{~S}(\mathbf{F})$ and 23S (G) rRNA of WT-like mutant B02.04 and r/tRNA insertion mutant F07.08. Expression is normalized with the expression of $g y r B$ using the formula $2^{\mathrm{C} q(g y r B)-\mathrm{Cq}(r R N A)}$. We performed a Student's t-test to compare the two conditions. *: $p$-value $<0.05 ;{ }^{* *}$ : $p$-value $<0.01$.

\section{Lp activates GCN2 signalling in the anterior midgut}

Small RNA produced by bacteria can be sensed by eukaryotic host's cells and modulate host's immune signalling (Koeppen et al., 2016; Moriano-Gutierrez et al., 2020; Ren et al., 2019). We hypothesized that products of the r/tRNAs loci may be microbial cues sensed by host cells to promote physiological adaptation. The general control nonderepressible 2 (GCN2) kinase can bind to and be activated by unloaded tRNAs (Wek et al., 1995) and rRNAs (Zhu and Wek, 1998). Moreover, the GCN2 pathway is one of the main pathways that allow eukaryotic cells to adapt to AA imbalance (Gallinetti et al., 2013). Finally, GCN2 is active in the gut of Drosophila 340 (Bonfini et al., 2021; Kim et al., 2021), where Lp is in close proximity to its host's cells (Fig. S3A). Therefore, we wondered if GCN2 may be a sensor and signalling intermediary between $r / t R N A$ from $L p$ and host physiological adaptation to an AA imbalanced diet.

345 We first tested whether Lp association regulates GCN2 activation in the larval gut. In Drosophila, the transcription factor ATF4 acts downstream of GCN2 and binds to recognition sites in the first intron of the gene 4E-BP (Thor in Drosophila) to activate its transcription (Kang et al., 2017). Kang and colleagues previously generated a transgenic line (4E-BPintrondsRed), which carries a fluorophore under the

350 transcriptional control of the first intron of $4 E-B P$. This reporter thus allows to visualize the pattern of activity of ATF4 downstream of GCN2 activation (Kang et al., 2017; Vasudevan et al., 2017). We used the 4E-BPintrondsRed reporter as a molecular readout to probe GCN2 activity in the larval midgut in response to $L p$ association. Fig. S3B shows the pattern of expression of the $4 \mathrm{E}-\mathrm{BP}$ intron $^{\mathrm{B}} \mathrm{R}$ Red reporter in dissected guts

355 of larvae fed an imbalanced diet (top panel) or a balanced diet (bottom panel), either GF (left panel) or Lp-associated (right panel). Similarly to what was previously reported (Kang et al., 2017), we observed 4E-BPintrondsRed reporter expression in the gastric caeca, the proventriculus and in the middle midgut, in a region known as the acidic zone (Overend et al., 2016). This pattern is conserved between GF larvae and Lp-

360 associated larvae. Conversely, the 4E-BPintrondsRed reporter is expressed in the anterior midgut specifically in Lp-associated larvae, while this signal is absent from GF guts (Fig. S3B, red squares and Fig. 3A). We confirmed by RT-qPCR that endogenous GCN2-dependent $4 E-B P$ expression is induced in the anterior midgut upon Lpassociation on an imbalanced diet (Fig. S3C). Interestingly, expression of the 4E$365 B^{\text {intron }}$ dsRed reporter in this region depends on the association with $L p$, but not on $A A$ imbalance as we observed it in larvae raised on either imbalanced diet (Fig. 3A, top 
panel) or balanced diet (Fig. 3A, bottom panel; quantification of the signal is shown in Fig. 3B). Lp can thus activate the 4E-BPintrondsRed reporter specifically in the anterior midgut, independently of dietary AA imbalance.

ATF4 is activated by elF2 $\alpha$, which can be phosphorylated by GCN2 but also by other kinases such as PERK (Teske et al., 2011). In order to test whether the 4E-

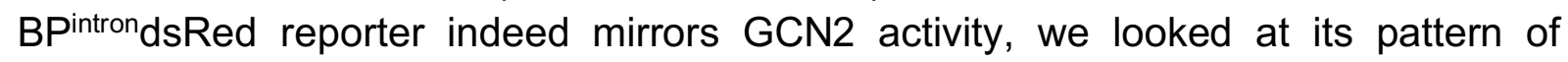
expression in a GCN2 knock-down background. Inhibition of GCN2 expression using 375 tissue specific in vivo RNAi (Dietzl et al., 2007) completely abrogates the activation of the $4 \mathrm{E}-\mathrm{BP}^{\mathrm{intron}} \mathrm{dsRed}$ reporter by $\mathrm{Lp}$ in the anterior midgut of larvae reared on imbalanced diet (Fig. 3C,D) or balanced diet (Fig. 3E,F). Therefore, our results establish that $L p$ promotes GCN2 activity in the anterior midgut of larvae, independently of AA imbalance. 
Figure 3

A.
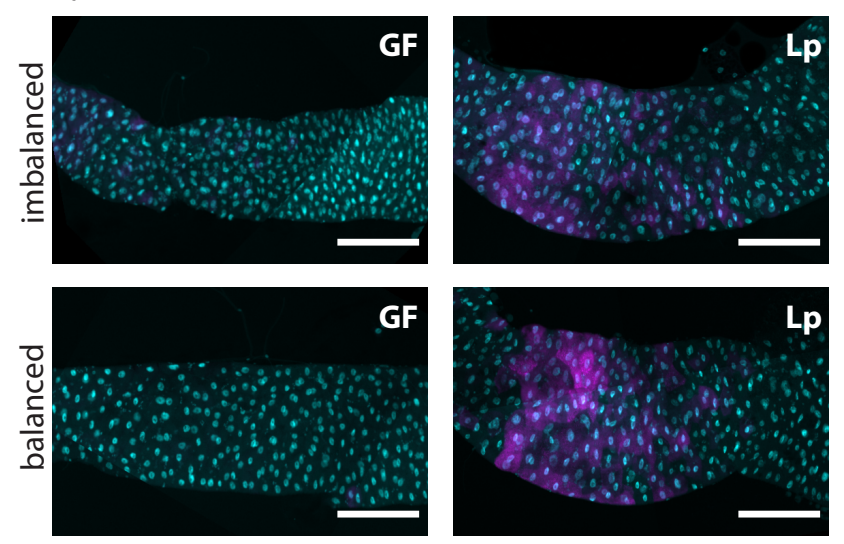

C.
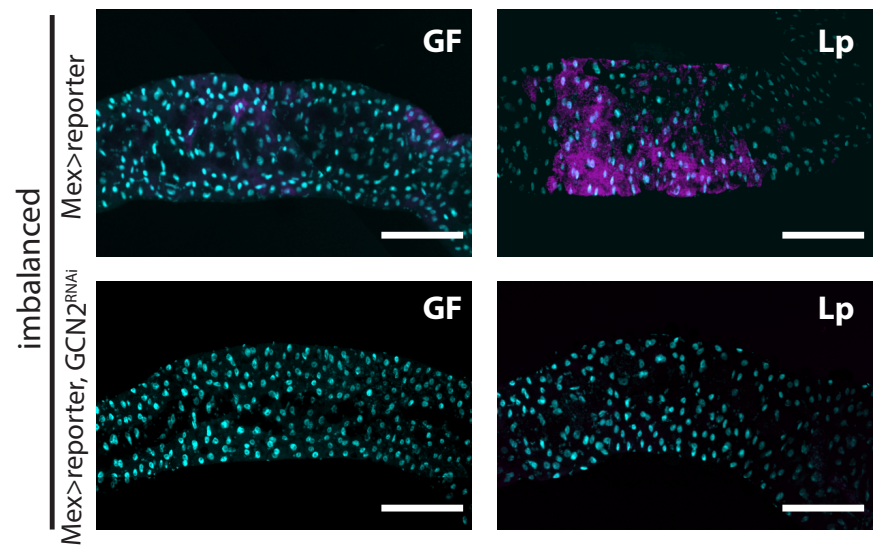

E.
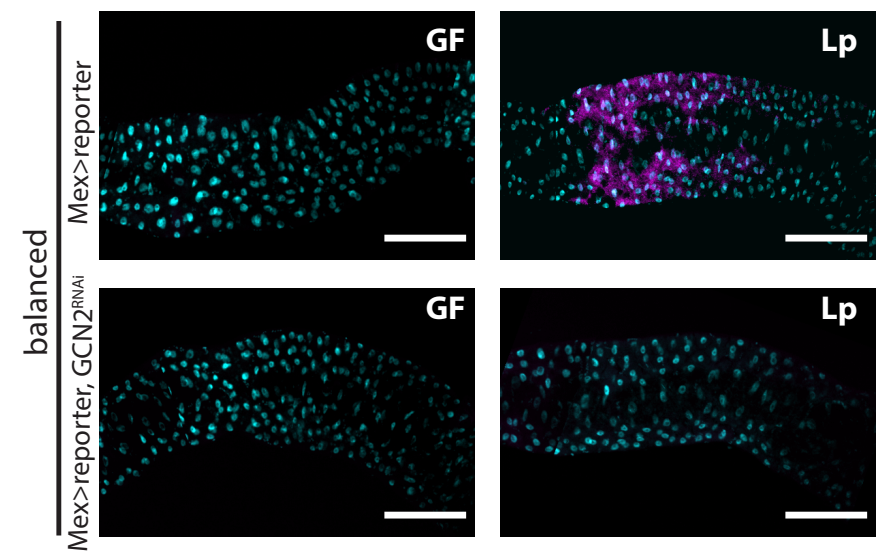

B.
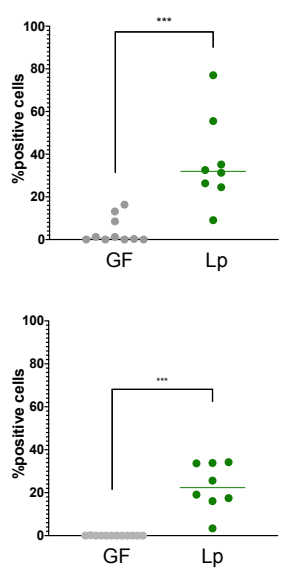

D.

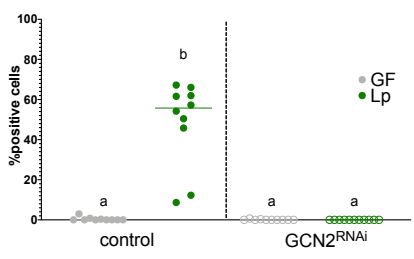

F.

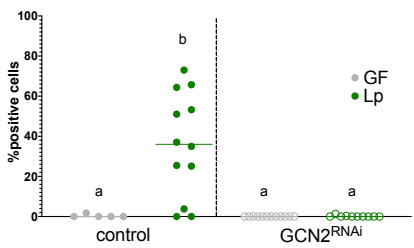

Fig. 3 Association with Lp activates GCN2 in the larval anterior midgut.

$(A, C, E)$ Representative pictures of the anterior midgut of 4E-BPintron dsRed larvae. Cyan: DAPI. Magenta: 4E-BPintrondsRed reporter. Scale bar: $200 \mu \mathrm{m}$. (B,D,F) Quantification of the signal: proportion of enterocytes positive for 4E-BPintrondsRed reporter's activity in the anterior midgut. Each dot represents an independent replicate. The bar shows the mean. (A) Larvae in GF conditions (left) or Lp-associated conditions (right) on imbalanced (FLY AA $-60 \%$ Val, top) or balanced (FLY AA, bottom) diet. Quantification of the signal in (B): GF larvae (grey) or Lp-associated larvae (green) fed an imbalanced 
bioRxiv preprint doi: https://doi.org/10.1101/2021.10.31.466661; this version posted December 21, 2021. The copyright holder for this preprint (which was not certified by peer review) is the author/funder, who has granted bioRxiv a license to display the preprint in perpetuity. It is made available under aCC-BY-NC 4.0 International license.

(top) or balanced (bottom) diet. We performed a Mann-Whitney test to compare the GF and Lpassociated conditions. ${ }^{* *}: p$-value $<0.01 .{ }^{* * *}$ : $p$-value $<0.001$. (C-E) Control larvae (Mex-Gal4 x 4EBPintrondsRed reporter) (top panel) and GCN2 knock-down (Mex-Gal4 x 4E-BPintrondsRed reporter, UAS$\mathrm{GCN} 2^{\mathrm{RNAi}}$ ) (bottom panel) in GF conditions (left) and Lp-associated conditions (right) fed an imbalanced $\operatorname{diet}($ FLY AA -60\% Val) (C) or a balanced diet (FLY AA) (E). Quantification of the signal in (D,F): GF larvae (grey circles) or Lp-associated larvae (green circles) fed an imbalanced diet (D) or a balanced diet (F). Filled circles: control condition (Mex-Gal4 x 4E-BPintrondsRed reporter). Empty circles: GCN2 knock-down (Mex-Gal4 x 4E-BPintron dsRed reporter, UAS-GCN2 ${ }^{\text {RNAi }}$ ). We performed a Kruskal-Wallis test followed by post-hoc Dunn's tests to compare all conditions together. a: the conditions are not significantly different from each other ( $p$-value $>0.05)$. $b$ : the condition is significantly different from other conditions ( $p$-value $<0.01$ ).

\section{Figure S3}

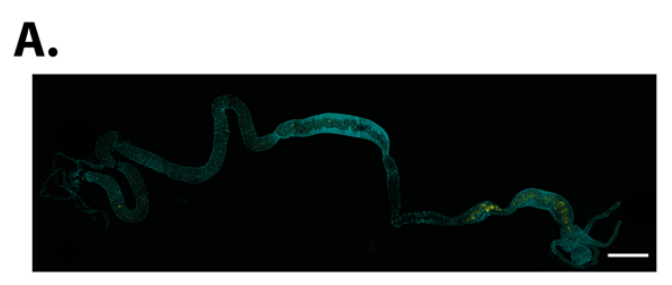

B.
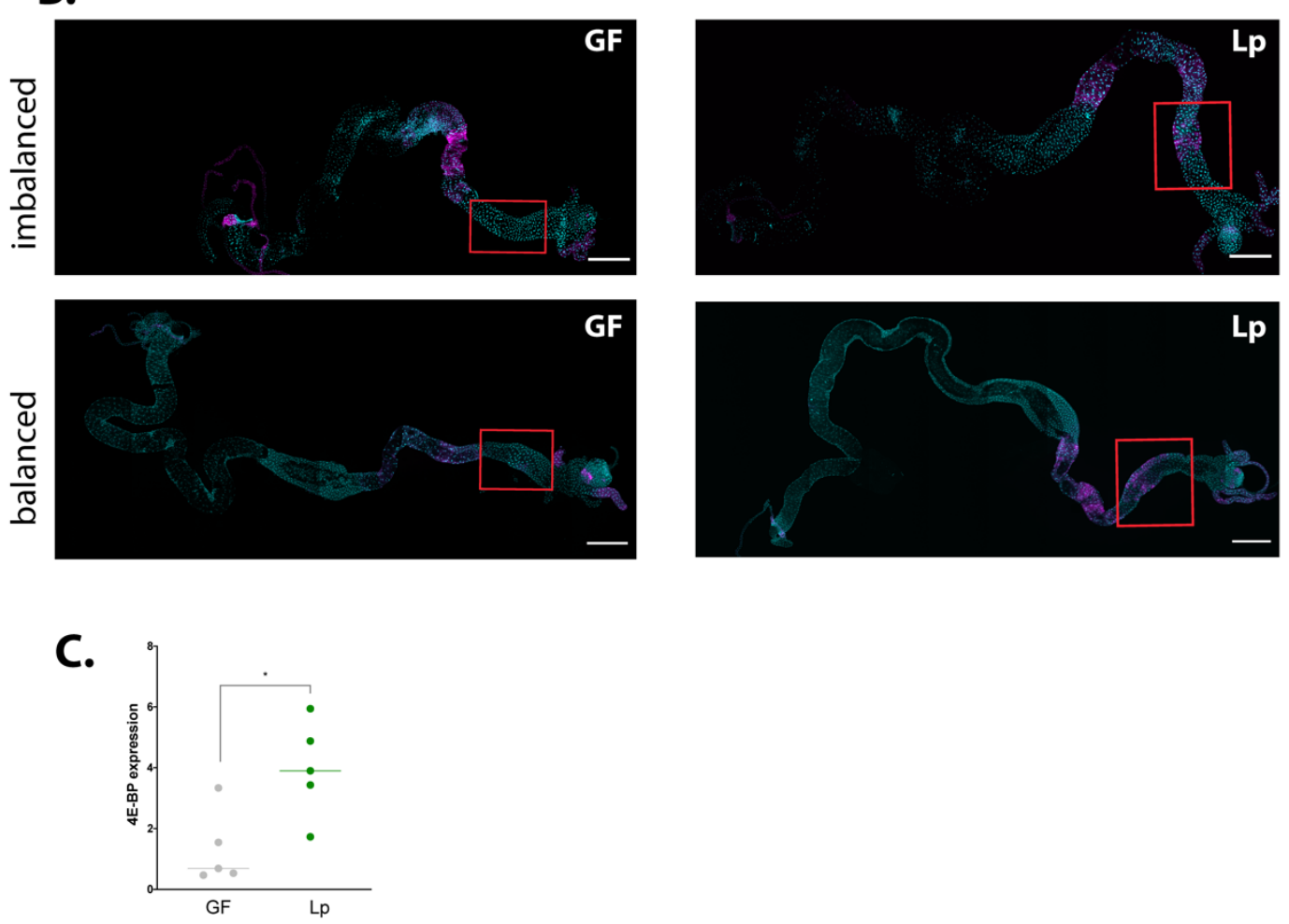

Fig. S3

(A) Representative picture of the full gut of a larva associated with Lp ${ }^{\text {GFP }}$. Cyan: DAPI. Yellow: GFP. Scale bar: $500 \mu \mathrm{m}$. (B) Representative pictures of the full gut of a GF larva (left panel) and a Lpassociated larva (right panel) fed an imbalanced diet (FLY AA -60\% Val, top panel) or a balanced diet (FLY AA, bottom panel). Cyan: DAPI. Magenta: 4E-BPintron dsRed reporter. The red squares show the region of the anterior midgut where $L p$ activates the $4 E-B P^{i n t r o n}$ dsRed reporter. Scale bar: $500 \mu \mathrm{m}$. (C) 
4E-BP expression in the anterior midgut of larvae reared on imbalanced diet (FLY AA -60\% Val) in GF conditions (grey) or Lp-associated conditions (green). Expression is normalized with the expression of rp49 using the formula $2^{\mathrm{Cq}(r p 49)-\mathrm{Cq}(4 E-B P)}$. We performed a Mann-Whitney test to compare the two conditions. *: p-value $<0.05$.

\section{Lp mutants for r/tRNA operon fail to activate GCN2 in the anterior midgut}

We then tested whether activation of GCN2 by Lp depends on its r/tRNA loci. To this end, we compared the induction of the 4E-BPintron $d s$ Red reporter in larvae associated with $\triangle \mathrm{op}_{\mathrm{r} / \mathrm{tRNA}}$ and in larvae associated with WT Lp. On an imbalanced diet, $\triangle \mathrm{op}_{\mathrm{r} / \mathrm{R} \mathrm{RA}^{-}}$ associated larvae show a reduced activation of GCN2 compared to WT-associated larvae (Fig. 4A,B). On a balanced diet, GCN2 activation in $\triangle \mathrm{op}_{\mathrm{r} / \mathrm{R} R \mathrm{R}}$-associated larvae is comparable to GCN2 activation in GF larvae, and significantly different from GCN2 activation in WT-associated larvae (Fig. 4C,D). Similarly, we observed that association with the r/tRNA insertion mutant F07.08 yields a reduction of GCN2 activation comparable to what we observed with $\triangle \mathrm{Op}_{\mathrm{r} / \mathrm{tRNA}}$ (on imbalanced diet: Fig. S4A,B; on balanced diet: Fig. S4C,D). The lesser difference observed on imbalanced diet might be due to longer association of the larvae with F07.08: indeed, in order to size-match the larvae, we collect them $24 \mathrm{~h}$ before the emergence of the first pupae, which is D6 after egg-laying for B02.04 and D8 after egg-laying for F07.08. To ensure comparable association time with the two mutants, we thus performed short-term association of GF larvae with B02.04 or F07.08: the larvae were reared GF, associated with B02.04 or F07.08 at D8 after egg-laying, and collected for dissection at D10 after egg-laying. In short-term association on an imbalanced diet, activation of GCN2 is significantly reduced in F07.08-associated larvae compared to B02.04-associated larvae (Fig. S4E,F).

Uncharged tRNAs and rRNA from eukaryotic cells are canonical activators of GCN2 kinase (Masson, 2019; Zhu and Wek, 1998). It is currently unknown whether bacterial r/tRNAs can activate GCN2 as well. We therefore tested this hypothesis by feeding purified bacterial tRNAs to GF larvae carrying the $4 \mathrm{E}-\mathrm{BP}^{\mathrm{intron}} \mathrm{dsRed}$ reporter. At the

440 highest dose tested $(625 \mu \mathrm{g})$, bacterial tRNAs significantly increase the expression of the reporter in the anterior midgut (Fig. 4E, F). This increase is comparable to the effect of feeding eukaryotic tRNAs to these larvae, though slightly inferior. However, the effect is minimal as compared to the association of larvae with Lp (Fig. S4G). Of note, $\mathrm{Lp}$ reaches a maximum of $\sim 2.5 \times 10^{9} \mathrm{CFUs} . \mathrm{mL}^{-1}$ in $\mathrm{HD}$ (Fig. 2D). Based on observations 445 made on other bacteria, this may yield $\sim 225 \mu \mathrm{g}$ of tRNAs in a $10 \mathrm{~mL}$ vial (Battley, 1988). This value is probably an underestimate, because tRNAs may accumulate during the stationary phase of $\mathrm{Lp}$; the value of $625 \mu \mathrm{g}$ thus appears to be within the physiological range of exposure of larvae. These results therefore suggest that Lp's tRNA may be direct activators of GCN2 in enterocytes. However, we cannot exclude 450 that Lp's rRNAs, or other yet unknown products of this loci or an indirect mechanism dependent on a functional Lp r/tRNA operon is at play. 


\section{Figure 4}
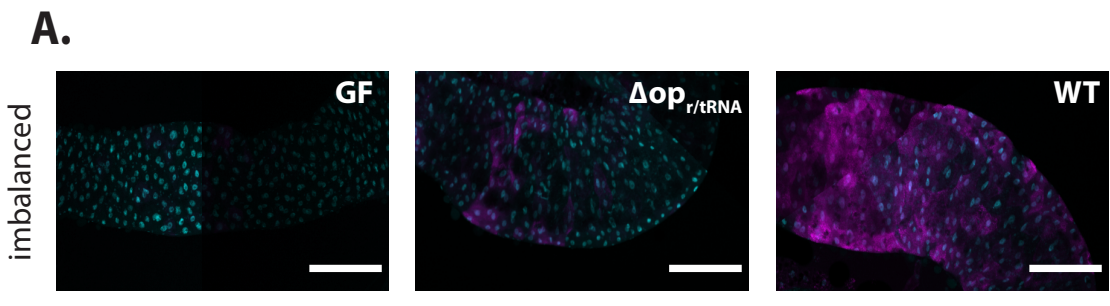

B.

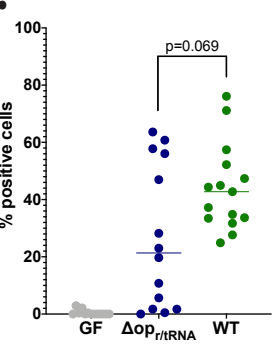

C.
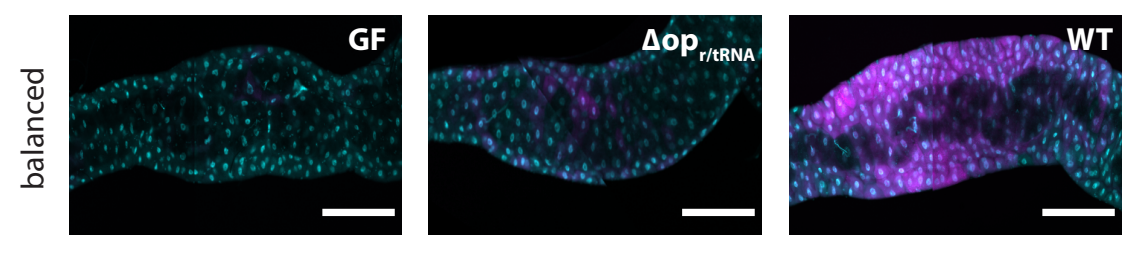

D.

E.

F.
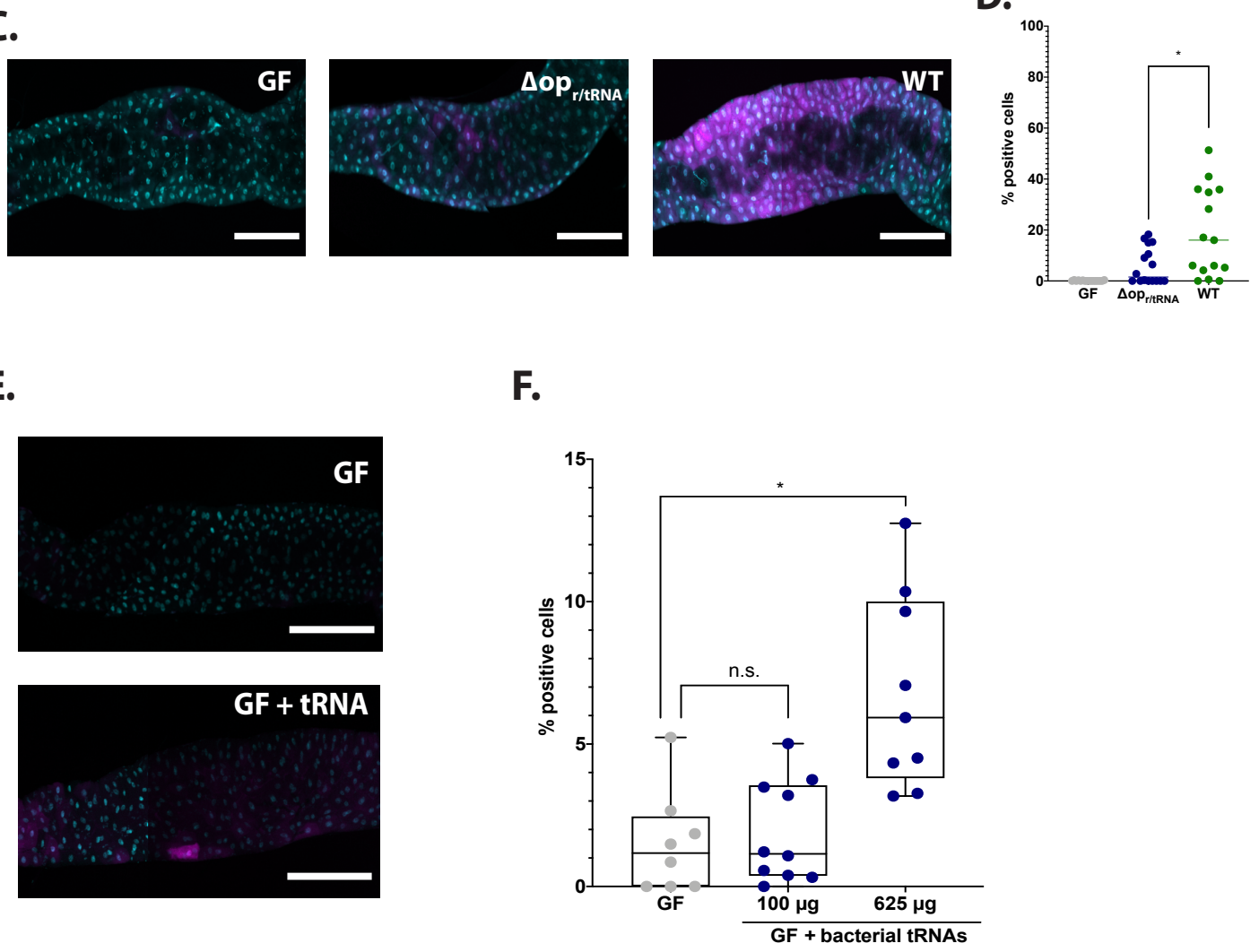

455 Fig. 4 Lp mutants for r/tRNA operon fail to activate GCN2 in the anterior midgut.

$(A, C)$ Representative pictures of the anterior midgut of 4E-BPintron dsRed larvae in GF conditions (left panels), in association with Lp $\triangle \mathrm{op}_{\mathrm{r} / \mathrm{RNA}}$ (middle panels) or in association with Lp WT (right panels). Cyan: DAPI. Magenta: 4E-BPintron dsRed reporter. Scale bar: $200 \mu \mathrm{m}$. (B,D) Quantification of the signal: proportion of enterocytes positive for 4E-BPintron dsRed reporter's activity in the anterior midgut of GF

460 larvae (grey), larvae associated with $L p \Delta \mathrm{op}_{\mathrm{r} \text { rtRNA }}$ (blue) and larvae associated with Lp WT (green). Each dot represents an independent replicate. The bar shows the mean. We performed Mann-Whitney test to compare association with $L p W t$ and association with $L p \Delta$ op rtRNA. $^{*}$ : $p$-value $<0.05$. (A) Larvae fed an imbalanced diet (FLY AA -60\% Val), signal quantified in (B). (C) Larvae fed a balanced diet (FLY AA), signal quantified in (D). (E) Representative images of 4E-BPintrondsRed GF larvae (top panel) and 4E-

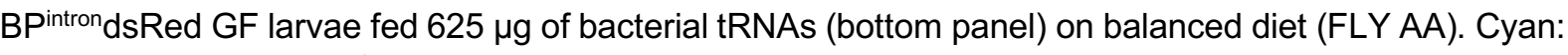
DAPI. Magenta: 4E-BPintrondsRed reporter. $(F)$ Quantification of the signal: proportion of enterocytes positive for 4E-BPintron dsRed reporter's activity in the anterior midgut of GF larvae (grey), GF larvae fed with increasing concentrations of bacterial tRNAs (blue) on balanced diet (FLY AA). We performed a 
bioRxiv preprint doi: https://doi.org/10.1101/2021.10.31.466661; this version posted December 21, 2021. The copyright holder for this preprint (which was not certified by peer review) is the author/funder, who has granted bioRxiv a license to display the preprint in perpetuity. It is made available under aCC-BY-NC 4.0 International license.

Kruskal-Wallis test followed by post-hoc Dunn's tests to compare each condition to GF. n.s.: nonsignificant. *: p-value<0.05.

Figure S4

A.
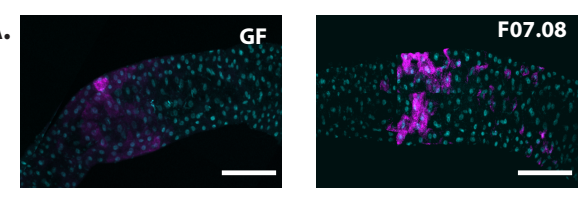

balanced diet

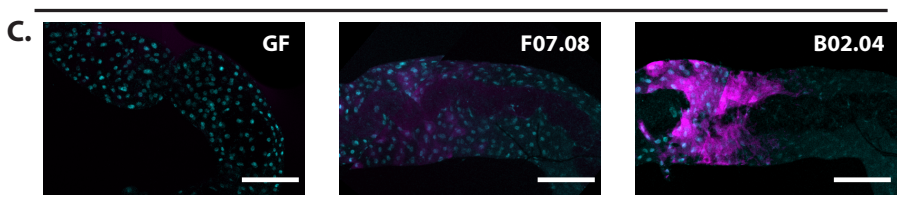

imbalanced diet, short-term association

E.
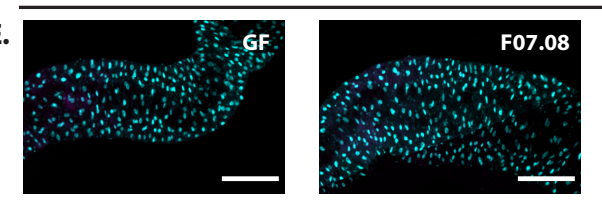
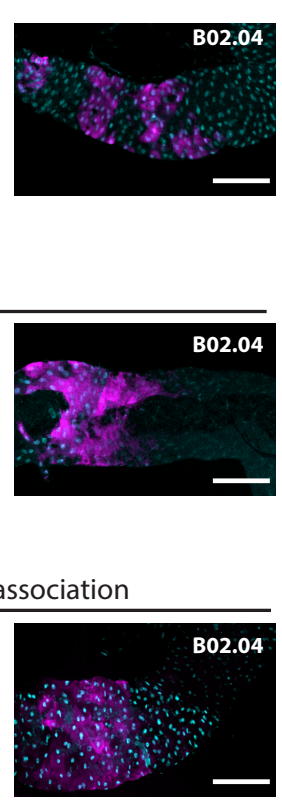

B. ${ }^{100}$

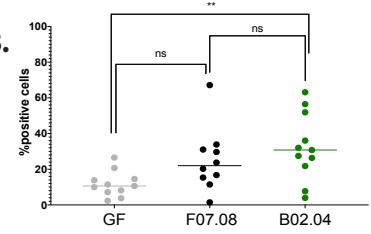

D.

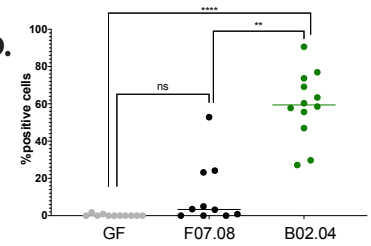

F.

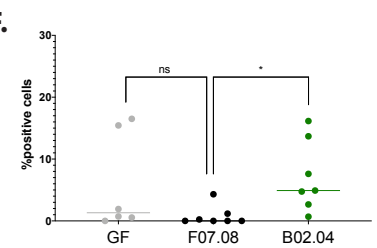

G.

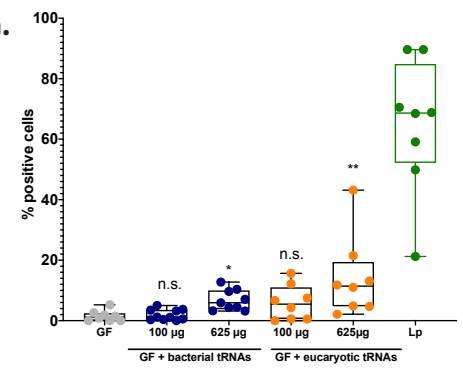

\section{Fig. 54}

$(A, C, E)$ Representative pictures of the anterior midgut of $4 \mathrm{E}-\mathrm{BP} \mathrm{Pitron}^{\mathrm{ds} R e d}$ larvae in GF conditions (left panels), in association with r/tRNA insertion mutant F07.08 (middle panels) or in association with WTlike insertion mutant B02.04 (right panels). Cyan: DAPI. Magenta: 4E-BPintron dsRed reporter. Scale bar: $200 \mu \mathrm{m}$. (B,D,F) Quantification of the signal: proportion of enterocytes positive for 4E-BPintrondsRed reporter's activity in the anterior midgut of GF larvae (grey), larvae associated with F07.08 (black) and larvae associated with B02.04 (black). Each dot represents an independent replicate. The bar shows the mean. We performed a Kruskal-Wallis test followed by post-hoc Dunn's tests to compare the conditions together. n.s.: non-significant. *: p-value $<0.05$. ${ }^{* *}$ : $p$-value $<0 ; 01$. (A) Larvae fed an imbalanced diet (FLY AA -60\% Val), signal quantified in (B). (C) Larvae fed a balanced diet (FLY AA), signal quantified in (D). (E) Larvae fed an imbalanced diet (FLY AA -60\% Val) upon short-term association with $L p$, signal quantified in (F). (G) Proportion of enterocytes positive for 4E-BPintron dsRed reporter's activity in the anterior midgut of GF larvae (grey), GF larvae fed with increasing concentrations of bacterial tRNAs (blue), yeast tRNAs (orange) or Lp-associated larvae (green) on balanced diet (FLY AA). We performed a Kruskal-Wallis test followed by post-hoc Dunn's tests to compare each condition to GF. n.s.: non-significant. *: p-value $<0.05$. **: p-value $<0.01$. ***: $p$-value $<0.001$. 


\section{Rescue of AA imbalance by Lp requires GCN2 in the larval midgut}

Therefore, association with Lp activates GCN2 in the anterior midgut of larvae in a r/tRNA loci-dependent manner. We thus sought to test whether GCN2 activation in enterocytes is necessary for $L p$ to rescue the developmental delay due to $A A$ imbalance. To this end, we specifically knock-downed GCN2 in enterocytes and followed the developmental timing of GF or Lp-associated larvae in AA-imbalanced conditions. On an imbalanced diet (-60\% Val), GCN2 knock-down in enterocytes causes a significant developmental delay in Lp-associated larvae compared to control larvae (Fig. 5A), a phenotype that we confirmed by using two other GCN2 RNAi lines (Fig. S5A,B) and by measuring the efficacy of RNA interference against GCN2 505 expression in the midgut (Fig. S5C). Of note, GCN2 knock-down in enterocytes does not alter colonization of the gut by Lp (Fig. S5D). Knocking-down GCN2 in the gut has no effect on larvae developing on an AA-balanced diet (Fig. 5B). Taken together, our results indicate that $G C N 2$ is necessary for $L p$ to rescue the developmental delay due to Val limitation. We wondered whether GCN2 is necessary to rescue AA imbalance

510 due to limitation in other AA. We decreased the amount of each EAA identified in Fig. 1 as most important for GF larvae (Ile, Lys, Thr and Trp) by $60 \%$ and measured the growth of larvae knocked-down for GCN2. We found that GCN2 is necessary for Lp to rescue the scarcity in lle or Thr, but not in Trp or Lys (Fig. 5C-F). Lp can synthesize Lys and Trp, but not Ile (Consuegra et al., 2020a); therefore, it seems that GCN2 is 515 necessary only when the limiting AA cannot be provided by Lp. Lp can produce Thr, but in limiting quantity (Consuegra et al., 2020a), which may explain why GCN2 is also necessary for $L p$ to rescue the delay due to Thr scarcity.

Lab-reared Drosophila are mostly associated with bacteria belonging to the families 520 Lactobacillales and Acetobacteraceae (Staubach et al., 2013). We tested whether a representative Acetobacteraceae strain, Acetobacter pomorum ${ }^{\mathrm{WJL}}$ (Ap) that promotes larval growth on oligidic nutrient-poor diet (Shin et al., 2011) and on complete holidic diet (Consuegra et al., 2020b) displays the same properties as Lp on AA imbalanced holidic diet. Ap rescues the developmental delay due to $A A$ imbalance to the same

525 extent as Lp (Fig. S6A). However, conversely to Lp, Ap's support to larval development upon Val limitation is independent of GCN2 expression in the gut (Fig. S6B). This is likely explained by the ability of Ap to produce Val and rescue the host's auxotrophy to Val (Consuegra et al., 2020a). Interestingly, Ap does not activate GCN2 in the anterior midgut of larvae on imbalanced diet (Fig. S6C,D) or balanced diet (Fig. S6E,F).

530 Therefore, the ability to activate GCN2 in the anterior midgut is not common to all symbiotic bacteria and a specific attribute of Lp-mediated benefit.

In Drosophila, it was previously reported that AA scarcity engages the GCN2 pathway in enterocytes of the midgut (Bonfini et al., 2021; Kim et al., 2021) but also in brain dopaminergic neurons (Bjordal et al., 2014) and in fat body cells (Armstrong et al., 
2014). Given that GCN2 activity in dopaminergic neurons of the larval brain promotes avoidance to EAA-deficient diets (Bjordal et al., 2014), we tested whether association with Lp alters larval food intake in our experimental conditions where larvae have no food choice to operate. We observed that Lp association does not alter food intake on imbalanced (Fig. S5E) or balanced diets (Fig. S5F). Moreover, knocking-down GCN2 expression in fat body cells has no impact on the development of Lp-associated larvae fed an imbalanced diet (Fig. S5G). These results suggest that the phenotype that we observe engages GCN2 in enterocytes, rather than in other tissues. Of note, although the target-of-rapamycin (TOR) pathway is another major factor supporting systemic

545 host growth in the fat body (Colombani et al., 2003) and in enterocytes (Redhai et al., 2020), it is not required in the fat body nor in enterocytes for Lp to rescue AA imbalance. However, it is important to support the growth of GF animals (Fig. $\mathrm{S} 5 \mathrm{H}, \mathrm{I}$ ).

Upon activation, GCN2 phosphorylates elF2 $\alpha$, the major subunit of the translation 550 initiation factor in eukaryotic cells (Donnelly et al., 2013). Phosphorylation of elF2 $\alpha$ inhibits translation, except for a subset of mRNAs including the transcription factor ATF4 which translation is increased (B'chir et al., 2013). ATF4 then activates the transcription of genes involved in stress response. In Drosophila, one of these genes is the elF4E-binding protein 4E-BP (Kang et al., 2017). 4E-BP activation promotes cap555 independent translation, which boosts Anti-Microbial Peptides (AMPs) production in the fat body (Vasudevan et al., 2017) and can alter the composition of the microbiota (Vandehoef et al., 2020). Therefore, we tested whether ATF4 and 4E-BP acting downstream of GCN2 are necessary for $L p$ to support larval development upon dietary AA imbalance. We observed that knocking-down ATF4 or 4E-BP in the enterocytes 560 delays the development of GF larvae on an imbalanced diet. However, such knockdowns do not affect the development of Lp-associated larvae like GCN2 knock-down does. ATF4 and 4E-BP are thus not required for $L p$ to support larval development upon dietary AA imbalance (Fig. 5G). Taken together, our results demonstrate the specific role of the GCN2 pathway in enterocytes to mediates Lp's support to larval 565 development despite dietary AA imbalance, independently of TOR and ATF4.

We then tested the interaction between GCN2 knock-down and Lp r/tRNA loci insertion mutants. As expected, control larvae reared on imbalanced diet in association with r/tRNA mutant F07.08 show a developmental delay compared to larvae associated 570 with WT-like B02.04 (Fig. 5H). On the contrary, larvae knocked-down for GCN2 in the enterocytes do not show a difference between association with F07.08 and association with B02.04 (Fig. 5I); in other words, the effect of the r/tRNA operon mutation is only observed when GCN2 is fully expressed. This suggests a functional interaction between products of the r/t RNAs loci in Lp and GCN2 in the larval gut and supports 575 the notion that Lp's influence on host adaptation to AA imbalance relies on a molecular dialog engaging symbiont r/tRNAs loci products and the host GCN2 kinase. 


\section{Figure 5}

A.

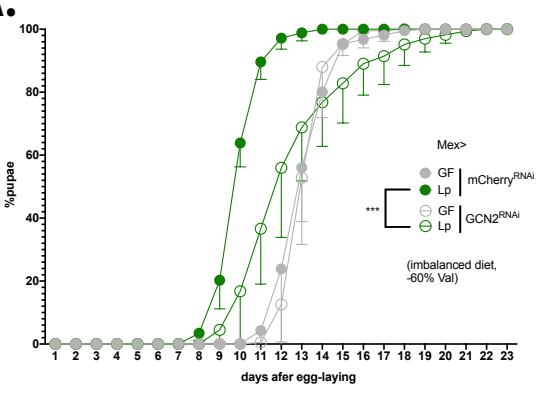

c.

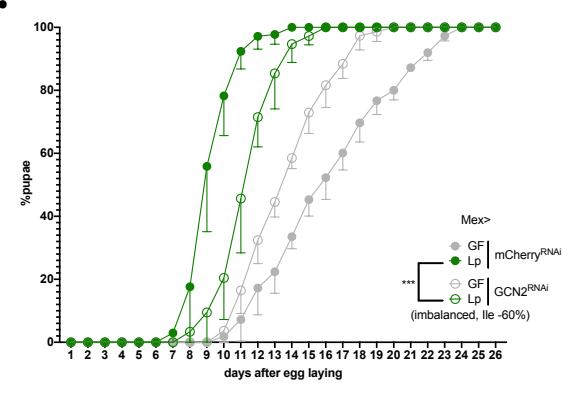

E.

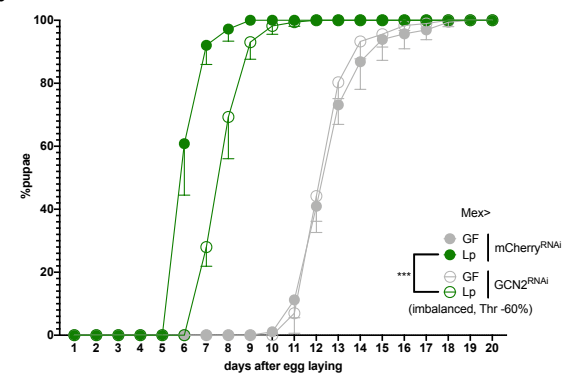

G.

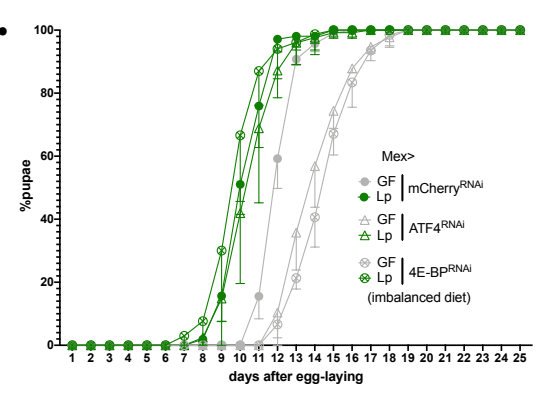

H.

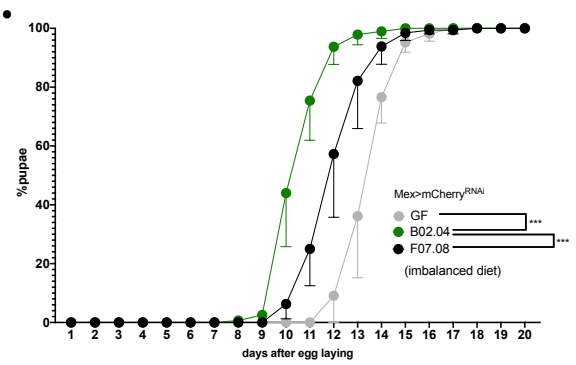

B.

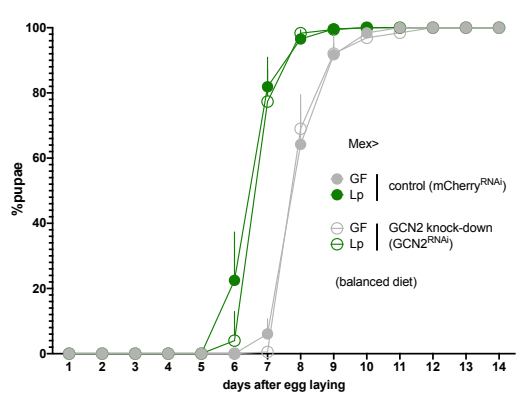

D.

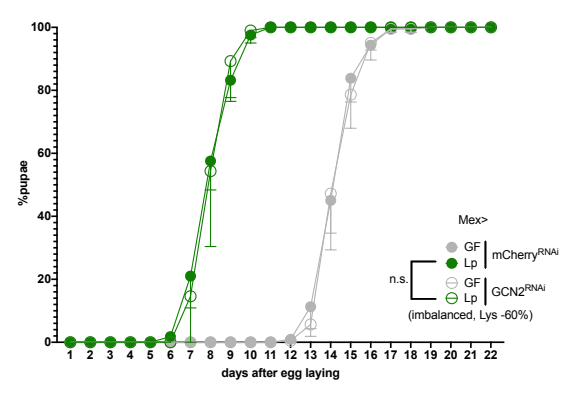

F.

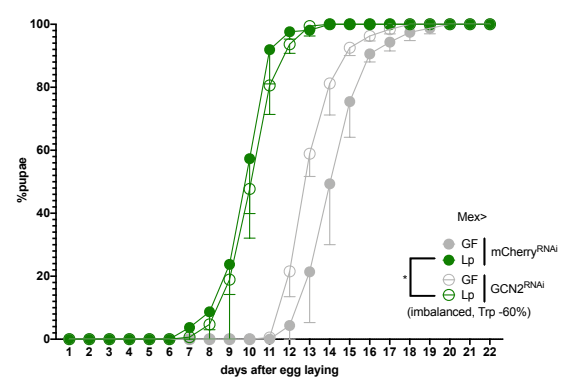

I.

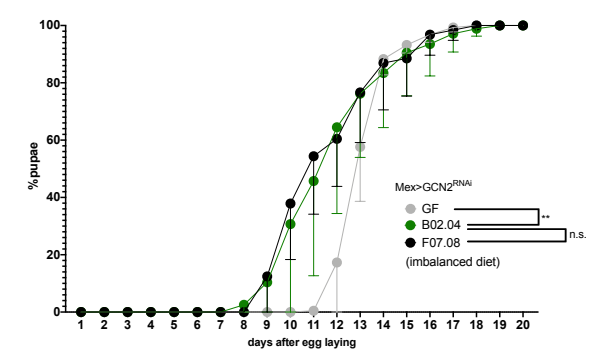

Fig. 5 Expression of GCN2 in the gut is necessary for Lp to rescue the delay due to AA imbalance. (A-G) Developmental timing of larvae raised in GF condition (grey circles) or Lp-associated conditions (green circles), in a control background (Mex>mCherry ${ }^{\mathrm{RNAi}}$, filled circles) or in a knock-down background 
bioRxiv preprint doi: https://doi.org/10.1101/2021.10.31.466661; this version posted December 21, 2021. The copyright holder for this preprint (which was not certified by peer review) is the author/funder, who has granted bioRxiv a license to display the preprint in perpetuity. It is made available under aCC-BY-NC 4.0 International license.

in enterocytes (empty circles). The graphs represent the total fraction of emerged pupae over time as a percentage of the final number of pupae. When indicated, we used a Cox proportional hazards model to compare the effect of $L p$ in the control background and in the GCN2 knock-down background. n.s.: non-significant. ${ }^{* *}$ : $p$-value $<0.01$. ${ }^{* * *}$ : $p$-value $<0.001$. (A-F) GCN2 knock-down in the enterocytes on imbalanced diet (-60\% Val) (A), balanced diet (B) or imbalanced diet: $-60 \%$ lle (C), -60\% Lys (D), -60\% $\operatorname{Thr}(\mathbf{E}),-60 \% \operatorname{Trp}(\mathbf{F})$. (G) ATF4 knock-down in the enterocytes (triangles) and 4E-BP knock-down in enterocytes (crossed circles) on imbalanced diet (-60\% Val). (H-I) Developmental timing of GF larvae (grey), larvae associated with r/tRNA mutant F07.08 (black) and larvae associated with WT-like mutant B02.04 (green) on imbalanced diet $(-60 \% \mathrm{Val})$ in a control background (Mex>mCherry $\left.{ }^{\mathrm{RNAi}}\right)(\mathrm{H})$ or in a

590 GCN2 knock-down in the enterocytes (Mex>GCN2 ${ }^{\text {RNAi }}$ (I). We used a Cox proportional hazards model to compare the effect of B02.04-association with F07.08-association and GF condition. n.s.: nonsignificant, "'*: p-value $<0.01,{ }^{* * *}$ : $p$-value $<0.001$. 


\section{Figure S5}

A.

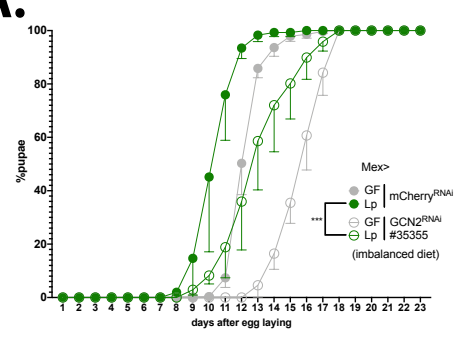

c.

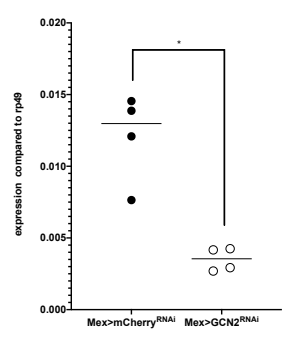

E.

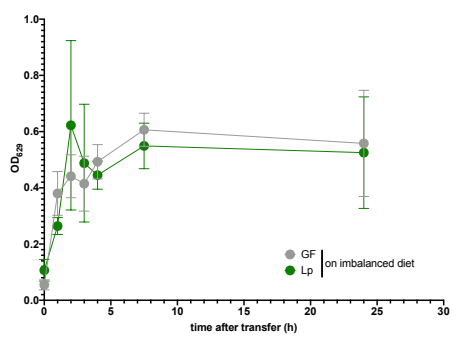

G.

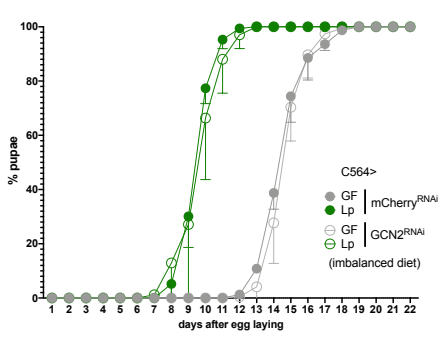

H.

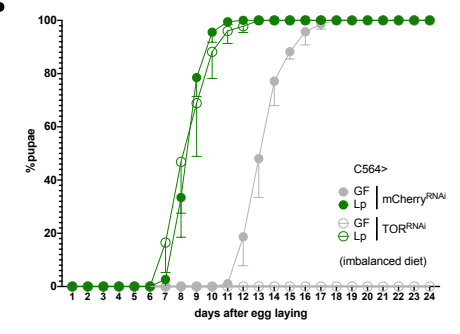

B.

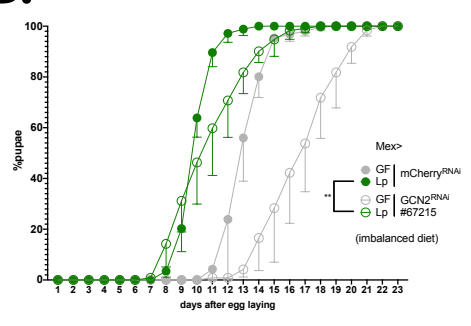

D.

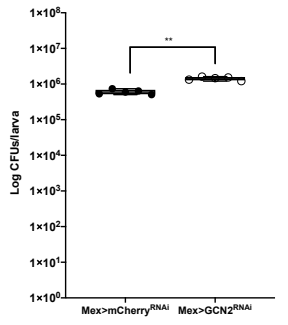

F.

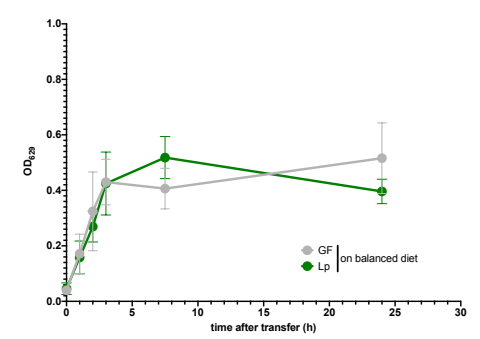

I.

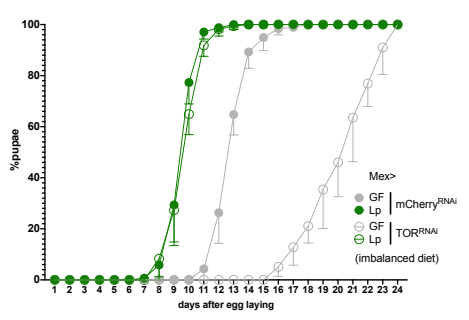

\section{Fig. S5}

(A-B) Developmental timing of GF larvae (grey) or Lp-associated larvae (green) from a control background (Mex>mCherry ${ }^{\mathrm{RNAi}}$, filled circles) or GCN2 knock-down larvae (Mex>GCN2 ${ }^{\mathrm{RNAi}}$, empty circles). The graph represents the total fraction of emerged pupae over time as a percentage of the final number of pupae. Larvae were reared on imbalanced diet (FLY AA -60\% Val). We used the lines \#BL 35355 (A) and \#BL 67215 (B). (C) Expression of GCN2 in the anterior midgut of control larvae (Mex>mCherry ${ }^{\mathrm{RNAi}}$ ) or GCN2 knock-down larvae (Mex>GCN2 ${ }^{\mathrm{RNAi}}$ ). Expression is normalized with the expression of $r p 49$ using the formula $2^{\mathrm{Cq}(r p 49)-\mathrm{Cq}(G C N 2)}$. We performed a Mann-Whitney test to compare 
bioRxiv preprint doi: https://doi.org/10.1101/2021.10.31.466661; this version posted December 21, 2021. The copyright holder for this preprint (which was not certified by peer review) is the author/funder, who has granted bioRxiv a license to display the preprint in perpetuity. It is made available under aCC-BY-NC 4.0 International license.

the two conditions, * $p$-value $<0.05$. (D) Colonization of the gut by Lp in control larvae (Mex>mCherry ${ }^{\text {RNAi, }}$ filled circles) or GCN2 knock-down larvae (Mex>GCN2 ${ }^{\text {RNAi }}$, empty circles) fed an imbalanced diet (FLY AA $-60 \%$ Val). The graph shows the quantity of Colony-Forming Units (CFUs) of Lp per larva. We performed a Mann-Whitney test to compare the two conditions. ${ }^{* *}: p$-value<0.01. (E-F) Food intake of GF larvae (grey) or Lp-associated larvae (green) reared on balanced diet (E) or imbalanced diet (F). Larvae were transferred on coloured food, and food intake was calculated as the Optical Density (OD) of the food ingested by the larvae over time. (G) Developmental timing of GF larvae (grey) or Lpassociated larvae (green) from a control background (C564>mCherry ${ }^{\mathrm{RNA} i}$, filled circles) or GCN2 knockdown in the fat body (C564>GCN2RNAi, empty circles). The graph represents the total fraction of emerged pupae over time as a percentage of the final number of pupae. Larvae were reared on imbalanced diet (FLY AA -60\% Val). (H, I) Developmental timing of GF larvae (grey) or Lp-associated larvae (green) from a control background (mCherry ${ }^{R N A i}$, filled circles) or TOR knock-down (TOR ${ }^{\text {RNAi, }}$

615 empty circles) in the fat body $(\mathrm{H})$ or in the enterocytes (I). The graph represents the total fraction of emerged pupae over time as a percentage of the final number of pupae. Larvae were reared on imbalanced diet (FLY AA -60\% Val). GF C564>TOR ${ }^{\mathrm{RNAi}}$ larvae did not reach pupariation. 
Fig. S6

A.

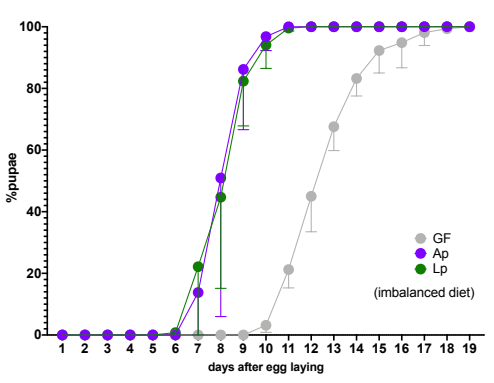

B.

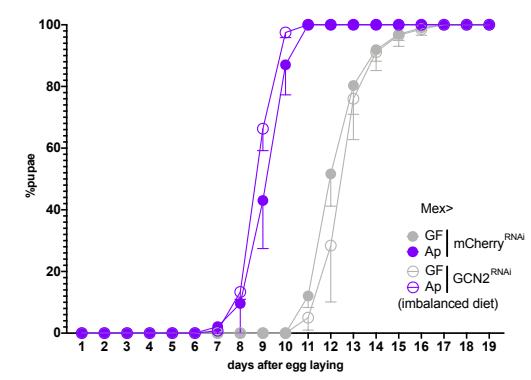

C.

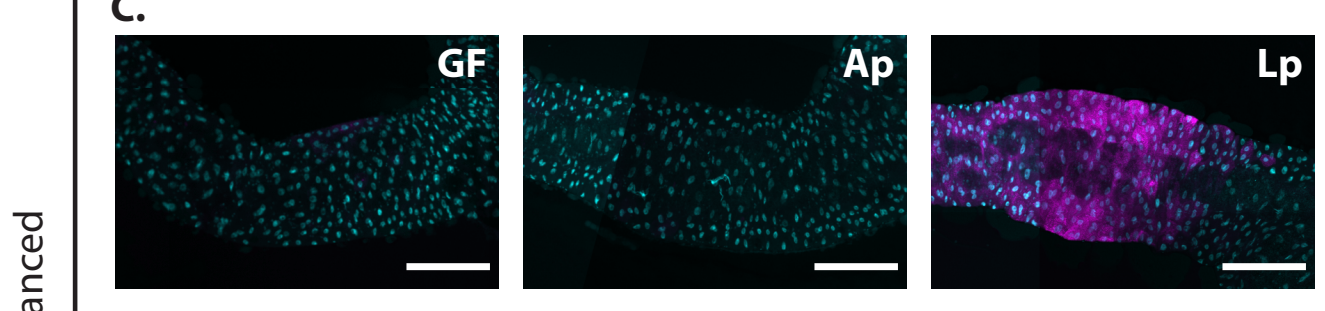

D.

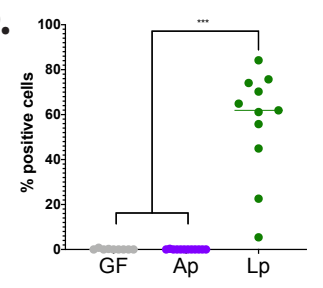

E.
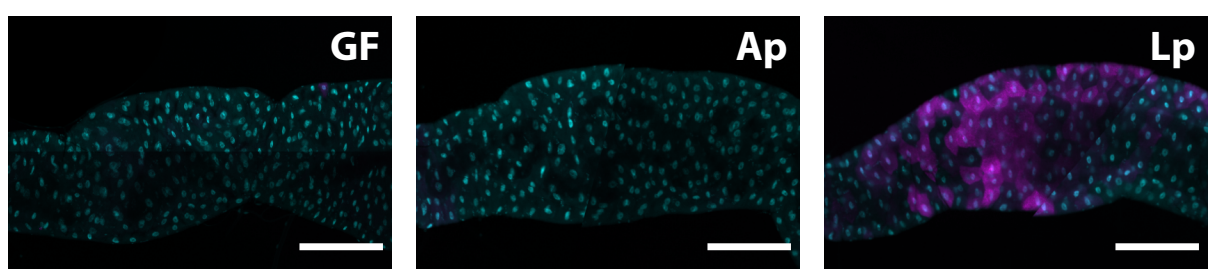

F.

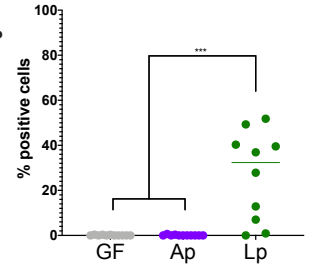

Fig. $\mathbf{S 6}$

(A) Developmental timing of larvae raised in GF condition (grey circles), Ap-associated conditions (purple circles) or Lp-associated conditions (green circles) on an imbalanced diet (FLY AA -60\% Val). The graph represents the total fraction of emerged pupae over time as a percentage of the final number of pupae. (B) Developmental timing of larvae raised in GF condition (grey circles) or Ap-associated conditions (purple circles), in a control background (Mex>mCherry ${ }^{\mathrm{RNAi}}$, filled circles) or in a GCN2 knockdown background (Mex>GCN2 ${ }^{\text {RNAi }}$, empty circles) on an imbalanced diet (FLY AA -60\% Val). The graph represents the total fraction of emerged pupae over time as a percentage of the final number of pupae. $(C, E)$ Representative pictures of the anterior midgut of $4 \mathrm{E}-\mathrm{BP}^{\text {intron }} \mathrm{dsRed}$ larvae. Cyan: DAPI. Magenta: 630 4E-BPintron dsRed reporter. Scale bar: $200 \mu \mathrm{m}$. Left panel: GF larva. Middle panel: Ap-associated larva. Right panel: Lp-associated larva. Larvae were reared on imbalanced diet (C) or balanced diet (E). (D,F) 
Quantification of the signal: proportion of enterocytes positive for 4E-BPintron dsRed reporter's activity in the anterior midgut. Each dot represents an independent replicate. The bar shows the mean. We performed a Kruskal-Wallis test followed by post-hoc Dunn's tests to compare GF and Ap to Lp. ${ }^{* *}$ : $p$ value $<0.001$. Larvae were reared on imbalanced diet (FLY AA -60\% Val) (D) or balanced diet (FLY AA) (F).

\section{The anterior midgut gut of Lp-associated larvae displays GCN2-dependent,} ATF4-independent and r/tRNA-dependent transcriptomic signatures of increased epithelium maturation and altered metabolic activity

We next wondered how GCN2 activation by products of Lp's r/tRNA operon may support larval growth on an AA imbalanced diet. To get a first handle on the molecular roots of this phenomenon, we sequenced the transcriptome of the anterior midgut of 645 third instar larvae raised on an imbalanced diet. We took advantage of the specificity of the phenotype to narrow down our analysis. Indeed, Lp rescues AA imbalance in a control background (Fig. 1), but not in an enterocyte-specific GCN2 knock-down background (Fig. 5). Moreover, although ATF4 is one of the downstream targets of GCN2, ATF4 knock-down in enterocytes does not prevent the rescue of AA imbalance

650 (Fig. 5G). Finally, rescue of AA imbalance depends on the presence of r/tRNA operons in Lp (Fig. 2 and Fig. 5H,I). Therefore, we took advantage of this series of phenotypical results informing on the key mediators to compare 6 different biological contexts: (1) GF or (2) Lp-associated control larvae, (3) Lp-associated Mex>GCN2RNAi larvae, (4) Lp-associated Mex>ATF4 ${ }^{\mathrm{RNAi}}$ larvae and control larvae associated with either (5) the

$655 \mathrm{r} / \mathrm{tRNA}$ insertion mutant F07.08 or (6) WT-like insertion mutant B02.04. We then searched for transcriptomic signatures (i.e. genes differentially expressed - DE) meeting the following criteria: (1) DE between Lp-associated control larvae and GF control larvae (2) DE (in the same direction as 1) between Lp-associated control larvae and Lp-associated GCN2 knocked-down larvae (3) NOT DE or with the inverse 660 direction of expression as 1) between Lp-associated control larvae and Lp-associated ATF4 knocked-down larvae (4) DE (in the same direction as 1) between B02.04associated larvae and F08.08-associated larvae (Fig. 6A).

Principal component analysis showed that Lp-associated samples are more distant of 665 the GF samples regardless of the genotype (Fig S7A). The microbial status is thus a major factor explaining transcriptomic variation. Moreover, among Lp-associated larvae, control larvae are more distant of ATF4 knock-down and GCN2 knock-down. This pattern was expected because ATF4 is downstream GCN2, and thus genes regulated by ATF4 should also be regulated by GCN2. However, ATF4 knocked-down 670 samples and GCN2 knocked-down samples are clearly separated, which indicates that our dataset may allow us to identify signatures of GCN2-dependent ATF4-independent gene regulation (Fig. S7A). Even though control larvae associated with B02.04 and F07.08 were closer, most samples were separated from each other by PC2 (Fig. S7B). We applied the four aforementioned selective filters (Fig. 6A) to our dataset and found 675104 genes consistently differentially expressed (25 up-regulated and 79 downregulated) by $L p$ in a GCN2-dependent, ATF4-independent and r/tRNA operondependent manner (Fig. 6B, C). These genes are listed in Table S1.

We then performed a Gene Ontology (GO) enrichment analysis to search for biological 680 functions enriched within the 25 up-regulated genes in Lp-associated larvae. We detected a significant enrichment for GO terms associated with organ morphogenesis, 
cell differentiation (different cell types: neurons, epithelial cells or stem cells) and cell proliferation (especially members of the Epidermal Growth Factor Receptor (EGFR) pathway) (Fig. 6D). These signatures suggest that Lp enhances the growth and maturation of the anterior midgut in a GCN2-dependent, ATF4-independent and r/tRNA operon-dependent manner. We then applied the same method to evaluate down-regulated genes, in search for biological functions repressed upon Lpassociation in a GCN2-dependent, ATF4-independent and r/tRNA operon-dependent manner. We found a significant enrichment in GO terms associated with mitochondrial respiration, resistance to oxidative stress and negative regulation of cell proliferation (Fig. 6E). Proliferative cells such as cancer cells, growing yeasts or embryonic cells tend to favour oxidative glycolysis and fermentation over mitochondrial respiration (in cancer cells, this phenomenon is known as the Warburg effect (Warburg, 1956)). Indeed, although mitochondrial respiration is more effective at producing ATP, 695 fermentation allows the synthesis of intermediates such as NADPH and ribose-5phosphate that are necessary for anabolism and cell growth. Moreover, fermentation allows to produce ATP at a faster rate (Lunt and Vander Heiden, 2011). Therefore, the decrease in genes responsible for mitochondrial respiration that we observe in the anterior midgut of Lp-associated larvae is in line with the hypothesis that the organ is ongoing remodelling and proliferation. Taken together, our transcriptomic analysis of the anterior midgut of larvae growing in an imbalanced diet allowed us to identify GCN2-dependent, ATF4-independent and Lp r/tRNA operon-dependent signatures evoking increase epithelium maturation and altered metabolic activity which would together support tissue growth and maturation. We posit that this phenomenon would also support the systemic growth of the animal. 
A

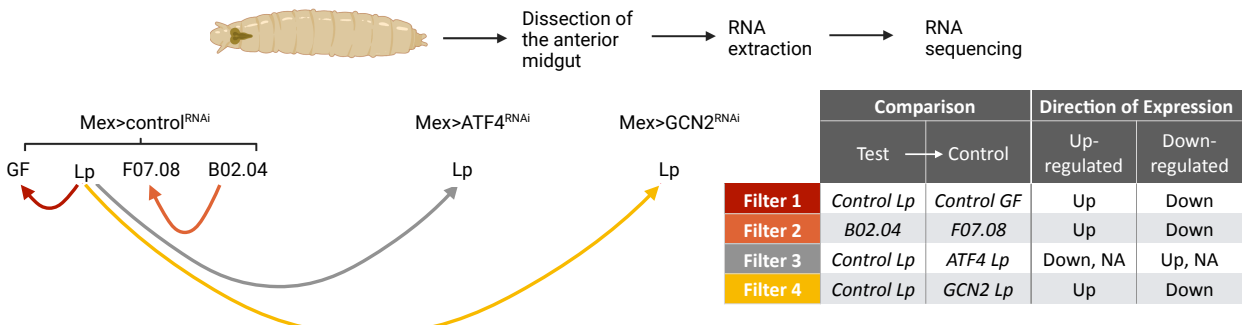

B

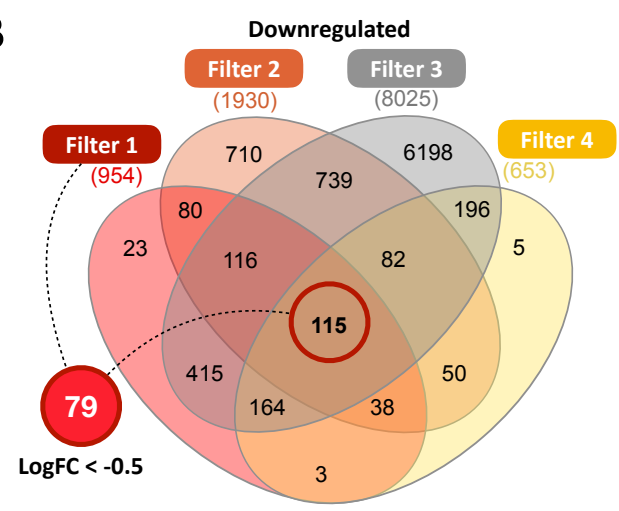

D

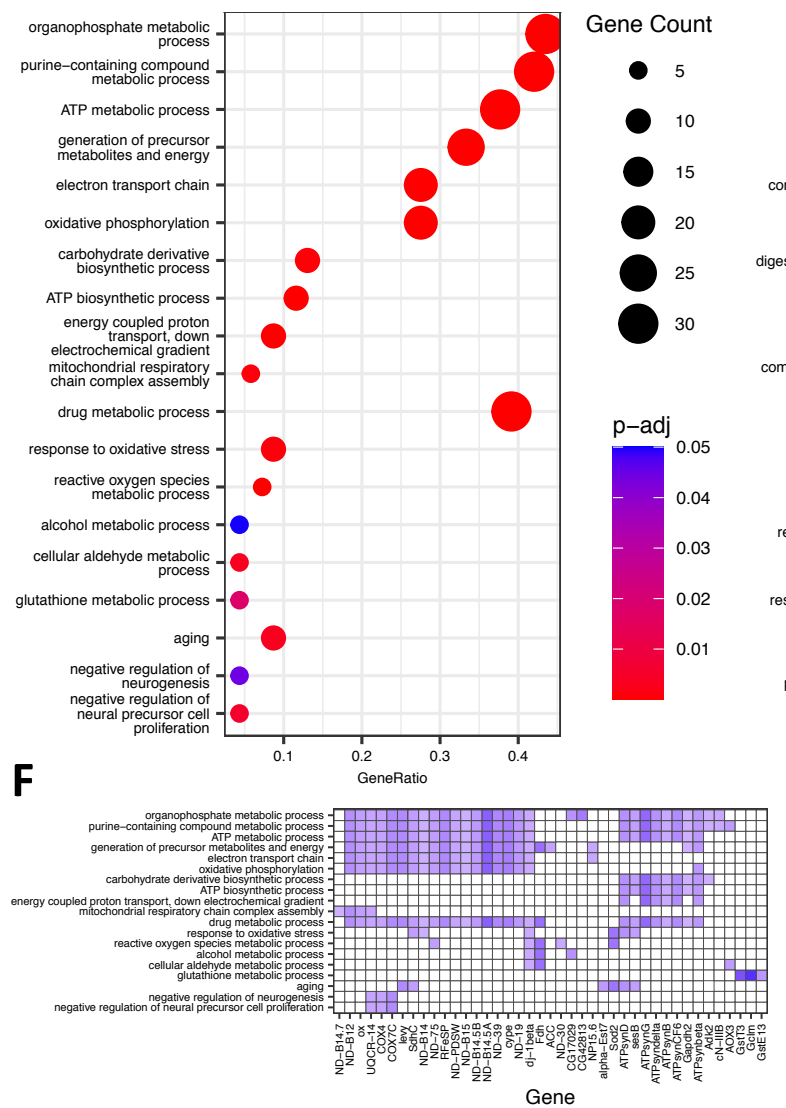

C

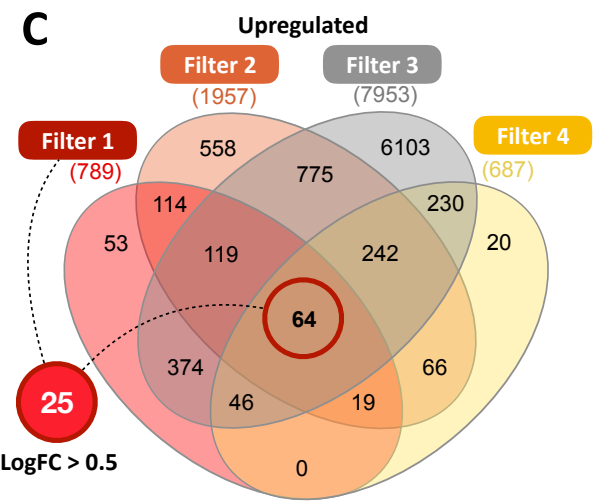

E

710 (A) Experimental design of the RNAseq strategy. We looked for genes differentially expressed (DE, red,

Fig. 6 The anterior midgut gut of Lp-associated larvae display transcriptomic signatures of remodelling and metabolic switch orange and yellow lines) or not differentially expressed (not DE, grey line) between conditions. Arrowheads indicate the control condition in each comparison. (B, C) Venn diagram showing the number of genes from each filter. We further selected genes from Filter 1 with a LogFC of at least 0.5 for genes up-regulated (B) and less than -0.5 for genes downregulated (C) for functional enrichment analyses. (D, E) Dot plot of Gene Ontology (GO) enrichment analysis of genes up-regulated (D) or down-regulated 
(E) by Lp-association in a GCN2-dependent, ATF4-independent and r/tRNA operon-dependent manner. The size of the dots represents the number of DE genes in each category, $x$-axis represents the gene ratio enrichment in comparison to the complete gene set of $D$. melanogaster; color indicates the significance in terms of $\mathrm{BH}$-adjusted p-values. (F,G) Denote the corresponding Log2 fold change (LogFC) plot from filter 1 (control Lp vs control GF) of genes belonging to the GO terms categories in (D) and (E) respectively.
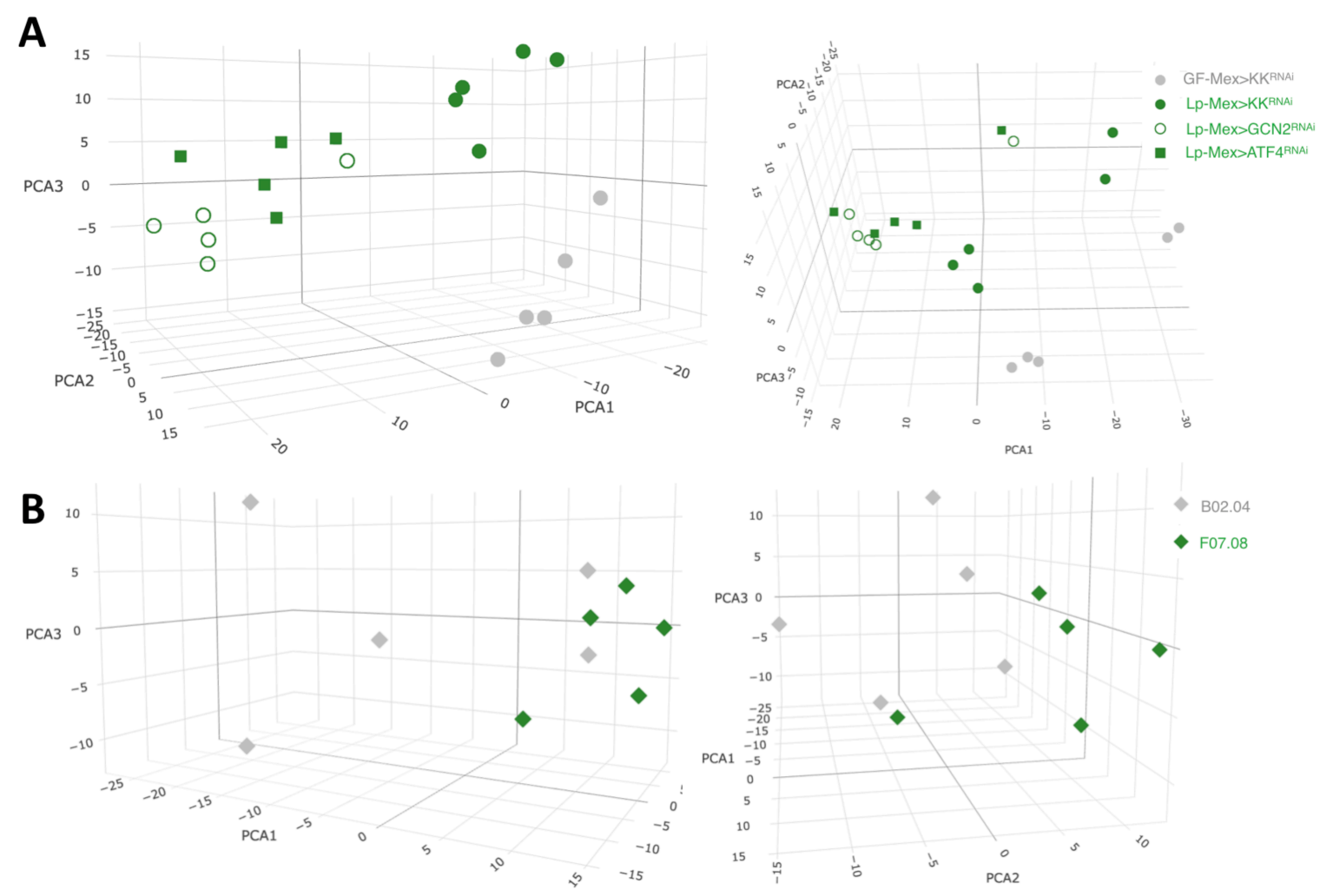

Fig. S7 3D Principal component analysis (PCA) plot showing the first 3 components, calculated with a variance stabilizing transformation of normalized counts from DESeq2. (A) PCA of the condition's Mex>control RNAi GF (grey circles) and Lp (green filled circles), Mex $>G C N 2^{\text {RNAi }}$ Lp (empty green circles) and Mex>ATF4 ${ }^{\text {RNAi }}$ Lp (green squares). (B) PCA of the conditions Mex $>$ control ${ }^{\text {RNAi }}$ associated with the WT-like insertion mutant B02.04 (grey diamonds) with the r/tRNA insertion mutant F07.08 (green diamonds).

\section{Discussion}

Symbiotic microbes can interact with their host through symbiotic cues: molecules produced by the symbiont that functionally interact with the host signalling pathways and influence various aspects of its physiology. Here, we reveal a novel mechanism supporting the symbiosis between Drosophila and its bacterial partner

740 Lactiplantibacillus plantarum. We show that Lp can rescue the developmental delay of its host due to $A A$ imbalance without providing the limiting AA. To understand how $L p$ promotes adaptation of its host to such dietary condition, we performed an unbiased 
genetic screen and showed that Lp operons encoding ribosomal and transfer RNAs are necessary to support host growth. We further showed that Lp activates the general control nonderepressible 2 (GCN2) signalling pathway in enterocytes of the anterior midgut. GCN2 activation depends on the presence of intact r/tRNAs-encoding operons in $L p$ and leads to a remodelling of the intestinal transcriptome suggesting tissue growth and maturation. GCN2 activation in the midgut is necessary to support growth on an imbalanced diet, but not on a balanced diet or when symbiotic bacteria can synthesize the limiting AA (e.g. Lp in the context of Lysine scarcity, or Ap upon Valine scarcity). These results therefore reveal a mechanism by which a symbiont supports its host physiological adaptation to a suboptimal nutritional environment by engaging the host kinase GCN2 in enterocytes, a previously underappreciated function of this signalling module in the host gut.

\section{How is GCN2 signalling engaged by Lp?}

We showed that GCN2 activation by Lp depends on the presence of the r/tRNA operons. GCN2 is known to be activated by several types of RNAs forming secondary 760 and tertiary structures: eukaryotic tRNAs cognate to several AA (Dong et al., 2000), free ribosomal eukaryotic RNAs (Zhu and Wek, 1998) and viral RNAs (Berlanga et al., 2006). Our results show that GCN2 can be activated by purified bacterial tRNAs, which make Lp's tRNAs interesting candidates. Of note, one of the mutants (D12.16) shows disruption of an operon that encodes only rRNAs. However, synthesis of tRNAs may

765 be regulated by ribosomal activity (Gourse et al., 1985); therefore, tRNAs production may be indirectly altered in D12.16 as well. Further work is needed to assess how the mutations that we identified impact the synthesis of each family of r/tRNA and to determine which of rRNA, tRNA, other RNA products of the loci or an indirect effect of the r/tRNA operon (for instance, through the translation rate in $L p$ that may be affected

770 by r/tRNA mutations) is responsible for r/tRNA operon-dependent activation of GCN2 by Lp. Of note, the other two candidates that we identified through the genetic screen might also be linked to r/tRNA production: C08.20 has a transposon insertion of the gene gnd2 that encodes a phosphogluconate dehydrogenase of the Pentose Phosphate Pathway (PPP). One product of the PPP is the 5-phosphoribosyl-a-1-

775 pyrophosphate (PRPP), which is a precursor for the biosynthesis of nucleotides (Kilstrup et al., 2005). It is thus possible that disruption of gnd2 in C08.20 might alter production of $r /$ tRNAs by the cells. B12.11 displays an insertion in the end of an operon encoding rsmC, Ip_0696 and Ip_sRNA01. rsmC encodes a 16S rRNA methyltransferase. Methylation of rRNA stabilizes ribosomes and improves translation 780 in other bacteria (Wong et al., 2013). Ip_0696 encodes a cytidine/deoxycytidylate deaminase, which catalyses conversion of cytidine into uridine. Ip_sRBA01 encodes the signal recognition particle (SRP), a small non-coding RNA which addresses membrane proteins to the membrane during their translation (Kuhn et al., 2017). Disruption of this operon may thus directly alter RNA production and/or ribosomal 785 function, which can negatively regulate r/tRNA synthesis (Gourse et al., 1985). Several studies have shown that small RNAs from bacteria can act as cues sensed by host 
790 Euprymna scolopes (Moriano-Gutierrez et al., 2020). Pseudomonas aeruginosa secretes tRNA-derived small RNAs that inhibit the immune response in cultured human cells and in the mouse lung (Koeppen et al., 2016). Lp is present to the ectoperitrophic space of the gut: it is not in direct contact with the enterocytes (Storelli et al., 2018). Therefore, we do not know how Lp's r/tRNAs may be delivered to enterocytes. $795 P$. aeruginosa's sRNAs and $V$. fischeri's SsrA were found inside extracellular vesicles (Koeppen et al., 2016; Moriano-Gutierrez et al., 2020). Interestingly, Lacticaseibacillus casei produces extracellular vesicles that contain r/tRNAs (Domínguez Rubio et al., 2017). Moreover, extracellular vesicles from Limosilactobacillus reuteri influence gut motility in mice (West et al., 2020) and extracellular vesicles from L. plantarumWCFS1 800 increase the expression of immunity genes in worms and cultured colonocytes (Li et al., 2017). Therefore, we propose a model whereby $L p$ would deliver r/tRNAs to Drosophila's enterocytes via extracellular vesicles. This model would explain why providing purified tRNAs directly to the larva does not fully recapitulate the effect of $L p$ on GCN2 activation, as if a vehicle (i.e. the extracellular vesicles) was needed.

We showed that GCN2 signalling is activated by Lp in enterocytes of the anterior midgut. This region of the midgut is of particular interest because it is located anteriorly to the acidic zone that kills a majority of Lp cells upon transit. It is thus the main region where enterocytes can interact with live Lp cells (Storelli et al., 2018). The pattern of 810 expression of the 4E-BPintron $\mathrm{dsRed}$ reporter slightly differs from the pattern observed by Kang and colleagues (Kang et al., 2017): upon AA scarcity, they observed activation of the reporter in the gastric caeca, the acidic region and the proventriculus like we did, but not in the anterior midgut. Those experiments were done in conventionally-reared larvae where the microbial status of the animals was not reported. We showed that 815 GCN2 activation in the anterior midgut is symbiont-dependent; this difference with our findings thus suggests that the larvae in Kang and colleagues' experiments were associated with bacteria that do not promote GCN2 activation in the anterior midgut, such as $A$. pomorum. An alternative explanation is that frequently flipped conventional fly stocks or crosses may carry very low microbial titers and therefore present GF-like

820 phenotypes. Of note, we could still observe expression of the 4E-BP ${ }^{\text {intron }} \mathrm{dsRed}$ reporter in the gastric caeca, the acidic region and the proventriculus upon GCN2 knock-down (whereas GCN2 knock-down completely abolishes 4E-BPintrondsRed expression in the anterior midgut, Fig. 3C-F). This suggests that other ATF4 activators such as PERK are active in the gastric caeca, the acidic region and the proventriculus. Importantly, 825 we observed activation of GCN2 by Lp in both contexts of imbalanced and balanced diet (Fig. 3A,B). We thus identified a GCN2 signalling activation mechanism which is AA-independent but bacteria-dependent. This differs from the canonical GCN2 activation relying on sensing unloaded eukaryotic tRNA upon AA scarcity. Previous studies have observed GCN2 activation in the Drosophila larval gut upon starvation 830 (Kang et al., 2017), upon lack of EAA in adults (Kim et al., 2021) and upon exposure 
to a high sugar/low protein diet in adults (Bonfini et al., 2021). These observations were made without control over the fly's microbiota, and it is thus difficult to conclude whether bacteria may be at play in these contexts (especially because microbial composition depends largely on diet composition (Lesperance and Broderick, 2020)). Studies have reported activation of GCN2 by pathogenic microbes: infection stimulates GCN2 in the gut, triggers a translational block (Chakrabarti et al., 2012) and promotes immune response through the ATF4-4E-BP axis (Vasudevan et al., 2017). The mechanism of activation of GCN2 by infection was not identified in these studies. Of note, Shigella infection causes GCN2 activation in HeLa cells, and it was proposed

840 that GCN2 is activated by AA depletion caused by the intracellular infection (Tattoli et al., 2012). However, this mechanism seems unlikely in our situation because Lp is not intracellular, and because association with Lp actually promotes physiological rescue of AA scarcity. In the light of our results, we suggest that GCN2 may also be activated by r/tRNA or small RNAs produced by pathogenic bacteria upon infection. These 845 studies and ours emphasize the role of GCN2 in the sensing of either pathogenic or symbiotic bacteria, in addition to its role in sensing uncharged eukaryotic tRNAs (Donnelly et al., 2013) and raise the question of the evolutionary history of GCN2 function: could GCN2 have primarily evolved as a pattern recognition receptor, i.e. a sensor for bacterial RNA later co-opted to sense AA scarcity indirectly through sensing uncharged eukaryotic tRNAs?

\section{How does GCN2 activation in the gut support improved systemic growth despite imbalanced nutrition?}

855 Our work shows that GCN2 expression in enterocytes is necessary for Lp's support to larval growth on an AA-imbalanced diet. However, overexpression of a constitutively active form of GCN2 in dopaminergic neurons represses growth on an AA-imbalanced diet by inhibiting food intake (Bjordal et al., 2014). These apparently contradictory results suggest that the action of GCN2 is organ-specific: in neurons, GCN2 may foster

860 adaptation to an imbalanced diet by prompting the larva to find a better food source; whereas in the gut, GCN2 may allow adaptation to an imbalanced diet through physiological changes of enterocytes. Studies in yeast or Drosophila GCN2 pathway have focused on the effector ATF4, which translation increases upon elF2 $\alpha$ phosphorylation by GCN2. Especially, Vasudevan and colleagues showed than the 865 GCN2-ATF4-4E-BP cascade yields the production of Anti-Microbial Peptides that help Drosophila fight infection (Vasudevan et al., 2017). Here, although we did indirectly observe activation of ATF4 (Fig. 3) and 4E-BP (Fig. S3C) upon Lp-association, this activation is not necessary for $L p$ to rescue the effects of $A A$ imbalance. Adaptation to AA imbalance may occur independently of elF2 $\alpha$, through other substrates of GCN2.

870 elF2 $\alpha$ is the only known substrate of GCN2 so far; however, Dang Do and colleagues showed that in the mouse liver, GCN2 does not regulate the same set of genes as PERK, another elF2 $\alpha$-kinase. This suggests that additionally to their common substrate elF2 $\alpha$, GCN2 and PERK have distinct substrates (Dang Do et al., 2009). 
875 (Grallert and Boye, 2007) and viral infection (Krishnamoorthy et al., 2008). Alternatively, support to growth despite AA imbalance by Lp may rely on elF $2 \alpha$ targets other than ATF4. ATF4-independent regulation of gene expression by elF2 $\alpha$ was described in Drosophila (Malzer et al., 2018), mice (Guo and Cavener, 2007) and cultured Mammalian cells (Harding et al., 2003; Wek and Cavener, 2007). Interestingly,

880 GCN2, but not ATF4, is necessary for adaptation of mice to a Leucine-depleted diet (Zhang et al., 2002). We took advantage of the specificity of the reported phenotype to find out how Lp may influence the physiology of the anterior midgut in a GCN2dependent, ATF4-independent and r/tRNA-dependent manner. Our transcriptomics data suggest that the anterior midgut of Lp-associated larvae shows increased tissue 885 growth and maturation and reduced mitochondrial respiration. GO terms associated with cell differentiation, cell proliferation (such as Epidermal Growth Factor receptor (EGFR) signalling) and morphogenesis are enriched in the Lp-associated conditions. This is in line with previous transcriptomic analyses in the midgut of adult flies, which showed that EGFR signalling and cell proliferation are stimulated by the gut microbiota 890 (Broderick et al., 2014). Moreover, genes related to mitochondrial respiration are more expressed in the GF condition when compared to Lp-associated larvae. This is suggestive of a metabolic switch from respiration to fermentation upon GCN2 activation by $L p$ that may favor host anabolism to increase the incorporation of cellular building blocks (such as amino acids, lipids, and nucleotides) into the host tissue (Lunt 895 and Vander Heiden, 2011). Importantly, transcriptomic remodelling upon GCN2 activation by $L p$ seems to be dependent on the presence of the $r / t R N A$ in $L p$ and independent of the expression of ATF4 in enterocytes. These signatures may help explain the phenotype of rescue of AA imbalance by Lp. In adults, midgut remodelling during reproduction promotes nutrient absorption and allows optimal fecundity (Ahmed 900 et al., 2020; Reiff et al., 2015). We propose that a similar mechanism may occur in larvae under Lp-association, through GCN2 activation dependent of the r/tRNA operon. Midgut remodelling via anabolism and maturation may improve nutrient absorption, including Valine, correcting dietary Valine scarcity and promoting systemic growth.

905

In conclusion, we showed that the symbiotic bacterium Lp can activate GCN2 in the enterocytes, likely through direct sensing of r/tRNAs as symbiotic cues. GCN2 activation in enterocytes induces a remodelling of the gut epithelium and a metabolic switch that may improve AA absorption and rescue the effects of AA imbalance on

910 larval growth. GCN2 is highly conserved across Eukaryotes (Donnelly et al., 2013), and is important for mouse adaptation to an AA-imbalanced diet (Anthony et al., 2004; Guo and Cavener, 2007; Laeger et al., 2016; Zhang et al., 2002). Our study therefore paves the way to testing whether the molecular dialogue between symbiotic bacteria and GCN2 described here is conserved in the animal kingdom.

\section{Material and Methods}




\section{Drosophila lines and breeding}

920 Drosophila stocks were maintained at $25^{\circ} \mathrm{C}$ with $12: 12-\mathrm{h}$ dark/light cycles on a yeast/cornmeal medium composed of $50 \mathrm{~g} . \mathrm{L}^{-1}$ of inactivated yeast, $80 \mathrm{~g} . \mathrm{L}^{-1}$ of cornmeal, $4 \mathrm{~mL} . \mathrm{L}^{-1}$ of propionic acid, $5.2 \mathrm{~g} . \mathrm{L}^{-1}$ of nipagin and $7.14 \mathrm{~g} . \mathrm{L}^{-1}$ of agar. All experiments were conducted in gnotobiotic flies derived from GF stocks. GF stocks were established as previously described (Combe et al., 2014) and maintained on yeast/cornmeal medium supplemented with antibiotics $(50 \mu \mathrm{g} / \mathrm{mL}$ of kanamycin, 50 $\mu \mathrm{g} / \mathrm{mL}$ of ampicilin, $10 \mu \mathrm{g} / \mathrm{mL}$ of tetracyclin and $5 \mu \mathrm{g} / \mathrm{mL}$ of erythromycin). We verified axenicity by grinding GF flies using a Precellys 24 tissue homogenizer (Bertin Technologies, Montigny-le-Bretonneux, France) and plating the lysate on ManRogosa-Sharp (MRS) Agar (Carl Roth, Karlsruhe, Germany) and LB Agar (Carl Roth, 930 Karlsruhe, Germany). We used $y w$ flies (BDSC \#1495) as a reference strain. The following lines were used: UAS-mCherry ${ }^{\mathrm{RNAi}}$ (BDSC \#35785), UAS-TOR ${ }^{\mathrm{RNAi}}$ (BDSC

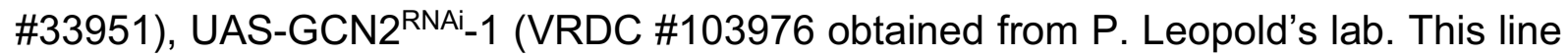
was used for all GCN2 knock-downs unless specified otherwise), UAS-GCN2 ${ }^{\text {RNAi-2 }}$ (BDSC \#35355), UAS-GCN2 ${ }^{\text {RNAi }} 3$ (BDSC 67215), UAS-ATF4 RNAi (VDRC \#109014), 935 UAS-4E-BPRNAi (VDRC \#36667), 4E-BPintrondsRed (obtained from H.D. Ryoo's lab), Mex1-Gal4 and C564-Gal4 from out stocks. We generated the line 4E-BPintrondsRed, UAS-GCN2 ${ }^{\text {RNAi }}$ by recombining the lines $4 \mathrm{E}-\mathrm{BP}^{\text {intron }}$ dsRed and UAS-GCN2 ${ }^{\mathrm{RNAi}}-1$. For RNA sequencing, we used UAS-GCN2 ${ }^{\text {RNAi-1 }}$ and UAS-ATF4 ${ }^{\mathrm{RNAi}}$, which are both KK lines from VDRC. In order to reduce transcriptional noise, we thus used the KK line

940 VDRC-60100 crossed to Mex1-Gal4 as the control condition.

\section{Holidic diets}

The holidic diets (HDs) were prepared following the protocol of Piper and colleagues 945 (Piper, 2017) at a total AA concentration of $10.7 \mathrm{~g} \cdot \mathrm{L}^{-1}$. We made two changes to Piper and colleagues' protocol: we used a lower concentration of sucrose $\left(5 \mathrm{~g} . \mathrm{L}^{-1}\right)$ because we noted that this concentration is the best for GF larvae: higher sucrose concentrations are toxic and slightly delay development of GF larvae (data not shown). Moreover, we omitted the conservatives (propionic acid or nipagin) because they can 950 alter bacterial growth. We worked in sterile conditions: tubes and egg-laying cages were UV-treated or autoclaved, and solutions were either autoclaved (first part containing agar, Leu, Ile, Tyr, sucrose, cholesterol and traces, as well as the acetate buffer solution) or filter-sterilized (stock solutions of EAA, NEAA, Glu, Cys, Vitamins, Nucleic Acids and Lipids precursors, Folate). For all experiments involving transposon 955 mutants, we supplemented the HD with Erythromycin $\left(5 \mu \mathrm{g} \cdot \mathrm{mL}^{-1}\right)$. HD was stored at $4^{\circ} \mathrm{C}$ for maximum one week before use.

\section{Bacteria and culture conditions}

960 We used the strain $\mathrm{Lp}^{\mathrm{NC} 8}$ of Lactiplantibacillus plantarum and the strain Ap ${ }^{\mathrm{WJ}}$ of Acetobacter pomorum. Conversely to other strains of $L$. plantarum, $L p^{N C 8}$ was not 
isolated from a fly but from grass silage (Axelsson et al., 2012); we used it because it is as growth promoting as fly isolates and it can be efficiently targeted for genetic modifications (Matos et al., 2017). $\mathrm{Lp}^{\mathrm{NC} 8}$ was grown overnight at $37^{\circ} \mathrm{C}$ without agitation in MRS Broth Medium (Carl Roth, Karlsruhe, Germany). The Lp mutant library was generated by random transposon insertion from $\mathrm{Lp}^{\mathrm{NC8}}$ (Matos et al., 2017). All Lp transposon insertion mutants were grown for 24h in MRS supplemented with Erythromycin at $5 \mu \mathrm{g} \cdot \mathrm{mL}^{-1}$. The L $\mathrm{p}^{\mathrm{GFP}}$ strain was generated from Lp ${ }^{\mathrm{WJL}}$ (Ryu et al., 2008; Storelli et al., 2018). L $p^{\text {GFP }}$ was grown for $24 \mathrm{~h}$ in MRS supplemented with 970 Chloramphenicol at $10 \mu \mathrm{g} \cdot \mathrm{mL}^{-1}$. Ap $\mathrm{pu}^{\mathrm{WJ}}$ was isolated from a fly's intestine (Ryu et al., 2008). Ap ${ }^{\text {WJL }}$ was grown for $24 \mathrm{~h}$ in Mannitol Broth composed of $3 \mathrm{~g} \cdot \mathrm{L}^{-1}$ of Bacto peptone (Becton Dickinson), 5 g.L-1 of yeast extract (Becton Dickinson) and 25 g. $\mathrm{L}^{-1}$ of D-mannitol (Carl Roth) in a flask at $30^{\circ} \mathrm{C}$ under $180 \mathrm{rpm}$ agitation.

\section{Developmental timing experiments}

GF flies were placed in a sterile breeding cage overnight to lay eggs on a dish of HD similar to the HD used for the experiment. At d0, we collected the eggs and placed them in the tubes containing the HD. Unless stated otherwise, each experimental 980 condition consisted in 5 tubes each containing 40 eggs. Eggs were then inoculated with $100 \mu \mathrm{L}$ of sterile PBS 1X (GF condition) or with $100 \mu \mathrm{L}$ of a culture of bacteria resuspended in PBS $1 \mathrm{X}$ (yielding $\sim 2 \times 10^{8} \mathrm{CFUs}$ of $\mathrm{Lp}$ and $\sim 10^{7} \mathrm{CFUs}$ of Ap per tube). For the Heat-Killed (HK) condition, the resuspension of Lp in PBS was incubated $3 \mathrm{~h}$ at $65^{\circ} \mathrm{C}$. After inoculation, the larvae were kept at $25^{\circ} \mathrm{C}$ with $12: 12$-h dark/light cycles.

985 The number of newly emerged pupae was scored every day until all pupae have emerged. The data are represented either as curves of pupariation over time or as the median time of pupariation (D50) calculated using a custom script on RStudio. We used the R package Coxme (Therneau and Grambsch, 2000) to make a Cox proportional hazard model adapted to curves of pupariation over time, replacing the

990 event "death" with the event "pupariation" (Rodrigues et al., 2021). Each experiment was performed in five independent replicates (one replicate consists in a tube containing 40 eggs as explained above). To compare the effect of $L p$ on development across different diets (e.g. balanced HD vs imbalanced HD), we tested whether the interaction between absence/presence of $L p$ and the diet was significant using the

995 model pupariation absence/presence of $L p$ * diet + (1|Replicate) (1|Replicate accounts for replicates as a random factor). When necessary, we used the FDR method to correct for multiple comparisons. To compare one treatment to other treatments (e.g. to compare the developmental timing of B02.04-associated larvae with GF larvae and F07.08-associated larvae), we performed a general linear hypotheses 1000 test (glht) from the package multcomp (Hothorn et al., 2008) using Tukey post hoc pairwise comparisons.

\section{Genetic screen}


1005 The genetic screen was performed in the same conditions as other Developmental timing experiments, but we used 20 eggs per condition in small tubes and one replicate per condition. Each condition consisted in the inoculation of one transposon insertion mutant. The screen was divided into four batches. For each batch, we calculated the D50 of each mutant, and the associated z-score. We then pooled the z-scores from 1010 the four batches and selected the ones above a threshold of 2.5. The 32 candidates were re-tested in a Developmental timing experiment of 5 replicates. We compared the candidates to the WT-like transposon insertion mutant B02.04 from the library (transposon inserted in an intergenic region downstream dnaJ).

\section{Mapping of insertions by Whole Genome Sequencing (WGS)}

The transposons inserted in the mutant's genomes are not bar-coded. To map them, we extracted the genomic DNA of each candidate using a kit UltraClean Microbial DNA isolation (MoBio, Jefferson City, Missouri, USA). Samples were quality-checked using

1020 a Qubit 4.0 HS DNA. To sequence genomic bacterial DNA, libraries were built using the Nextera DNA Flex Library Prep (Illumina, San Diego, California, USA) starting from $500 \mathrm{ng}$ of DNA (except for 2 samples for which $350 \mathrm{ng}$ and $280 \mathrm{ng}$ were used) and following the provider's recommendations. The 17 dual-indexed libraries were pooled in an equimolar manner and sequenced on a paired-end mode $(2 \times 75 \mathrm{bp})$ using a

1025 NextSeq500 Illumina sequencer and a mid-output run. More than 155M of reads were obtained for the run generating between $7 \mathrm{M}$ to $12 \mathrm{M}$ of reads by sample. Data were analyzed using Galaxy (Afgan et al., 2016). Briefly, for each mutant, we filtered all pairs of reads which had one of the two reads mapped on the transposon sequence. We gathered the paired reads and mapped them on the genome of $\mathrm{Lp}^{\mathrm{NC} 8}$ (Axelsson et al.,

10302012 ) to identify the region in contact of the transposon. The genome of L $\mathrm{p}^{\mathrm{NC}}$ contains five operons encoding r/tRNAs that share high sequence similarities. Therefore, sequencing did not allow us to identify in which operon the insertion took place. We thus used operon-specific PCR to identify in which operon the transposon was inserted for each mutant. For each mutant, we used two primers specific of the transposon

1035 (OLB215 and OLB 221) and one primer specific of each r/t RNA operon (op1, op2, op3, op4 and op5). The sequences of all primers used in this study can be found in Table S2.

\section{Construction of Lp $\Delta O \mathrm{op}_{\mathrm{r} / \mathrm{tRNA}}$}

We deleted the operon encoding rRNA 5S, 16S and 23S as well as tRNAs for Ala, Ile, Asn and Thr that was independently identified in two insertion mutants from the genetic screen (C09.09 and F07.08, see Fig. 2G). We used homology-based recombination with double-crossing over as described in (Matos et al., 2017). Briefly, Lp ${ }^{\text {NC8's }}$

1045 chromosomic DNA was purified with a kit UltraClean Microbial DNA isolation (MoBio, Jefferson City, Missouri, USA). The regions upstream and downstream of the operon were then amplified using Q5 High-Fidelity 2X Master Mix (New England Biolabs, Ipswich, Massachusetts, USA) with the following primers that contain overlapping 
regions with the plasmid pG+host9 (Maguin et al., 1996) to allow for Gibson Assembly: tRNAop_1, tRNAop_2, tRNAop_3 and tRNAop_4 (Table S2). The PCR fragments were inserted into the plasmid pG+host9 digested with Pstl (New England Biolabs, Ipswich, Massachusetts, USA) by Gibson Assembly (New England Biolabs, Ipswich, Massachusetts, USA) and transformed into E. coli TG1. The plasmid was purified and transformed into E. coli GM1674 dam dcm $^{-}$(Palmer and Marinus, 1994) to demethylate

1055 the DNA. The plasmid was then purified and transformed into $L \mathrm{p}^{\mathrm{NC}}$ by electroporation. Transformants were grown in MRS supplemented with Erythromycin at $5 \mu \mathrm{g} \cdot \mathrm{mL}^{-1}$ at $42^{\circ} \mathrm{C}$, which does not allow replication of the plasmid, in order to select integration of the plasmid into the chromosome by crossing-over. Plasmid excision associated with deletion of the operon by a second crossing-over event was then obtained by sub-

1060 culturing Lp in absence of Erythromycin. The deletion was screened by colony PCR and confirmed by sequencing using the primers tRNAop_5 and tRNAop_6 (Table S2).

\section{Microscopy}

1065 4E-BPintrondsRed larvae were reared on HD as described for Developmental timing experiments. We collected them at pre-wandering mid-L3, 1 day before the emergence of the first pupae (typically D5 after egg-laying (AEL) for Lp-associated larvae on balanced diet, D6 AEL for Lp-associated larvae on imbalanced diet or GF larvae on balanced diet, D7 AEL for GCN2 knocked-down Lp-associated larvae on imbalanced

1070 diet, D10 AEL for GF larvae on imbalanced diet, D6 AEL for B02.04-associated larvae on imbalanced diet and D8 AEL for F07.08-associated larvae on imbalanced diet). For short-term association, GF larvae were reared on imbalanced diet until D8 AEL, associated with B02.04 and F07.08 as previously described and collected at D10 AEL. Larvae were dissected in PBS 1X. The guts were fixed in paraformaldehyde (PFA) $4 \%$

1075 in PBS 1X 1h at room temperature, washed in PBS 1X, washed three times in PBS Triton $0.2 \%$, washed in PBS1X and mounted in ROTI®Mount FluorCare DAPI (Carl Roth, Karlsruhe, Germany). Pictures were acquired with a confocal microscope Zeiss LSM 780 (Zeiss, Oberkochen, Germany). We analysed the images on Fiji (Schindelin et al., 2012) using a custom macro: the macro identifies the reporter-positive regions

1080 above a defined threshold in the anterior midgut, and count the number of DAPIpositive particles inside these regions. It then counts the total number of DAPI-positive particles in the anterior midgut and uses it for normalization.

\section{Bacterial growth on HD}

1085

Microtubes containing $400 \mu \mathrm{L}$ of imbalanced HD were inoculated with $\sim 10^{6}$ CFUs of each candidate mutant. 5 L1 GF larvae were added to each tube, and the tubes were incubated at $25^{\circ} \mathrm{C}$. Each day, 3 samples per condition were collected for CFUs counting: we added $600 \mu \mathrm{L}$ of sterile PBS $1 \mathrm{X}$ and grinded them using a Precellys 24

1090 tissue homogenizer (Bertin Technologies, Montigny-le-Bretonneux, France. Settings: $6000 \mathrm{rpm}, 2 \times 30 \mathrm{~s}, 30 \mathrm{~s}$ pause). The homogenates were diluted at the appropriate concentration and plated on MRS Agar using an Easyspiral automatic plater 
(Interscience, Saint-Nom-la-Breteche, France). Plates were incubated at $37^{\circ} \mathrm{C}$ for $48 \mathrm{~h}$, and the number of CFUs was assessed using an automatic colony counter Scan1200 (Interscience, Saint-Nom-la-Breteche, France) and its counting software.

\section{Colonization of the larval gut}

Larvae were reared on imbalanced diet as for Developmental timing experiments. 6 1100 days AEL, larvae were collected, surface-sterilized in ethanol $70 \%$ and grinded using a Precellys 24 tissue homogenizer (Bertin Technologies, Montigny-le-Bretonneux, France. Settings: $6000 \mathrm{rpm}, 2 \times 30 \mathrm{~s}, 30 \mathrm{~s}$ pause). The CFUs were then counted as described above.

\section{5 tRNAs feeding}

$4 \mathrm{E}-\mathrm{BP} \mathrm{intron}^{\mathrm{a}} \mathrm{R}$ Red larvae were reared on balanced diet as for Developmental timing experiments. At d0, d2, d4 and d5 AEL, the tubes were supplemented with $50 \mu \mathrm{L}$ of a solution of tRNAs dissolved in Millipore water to reach a total concentration in the tube 1110 of 5,25 and $125 \mu \mathrm{g} \cdot \mathrm{mL}^{-1}$. GF controls were supplemented with the same volume of Millipore water. We purchased the purified tRNAs at Sigma-Aldricht (Saint-Louis, Missouri, USA; bacterial tRNAs from Escherichia coli 10109541001, eukaryotic tRNAs from yeast 10109517001). Larvae were dissected 6 days AEL and treated as described above.

\section{Food intake experiments}

Larvae were reared on holidic diet as described for Developmental timing experiments. Larvae were collected 1 day before the emergence of the first pupae and placed on

1120 holidic diet containing Erioglaucine disodium salt (Sigma-Aldrich, Saint-Louis, Missouri, USA) at $0.8 \%$. Every hour, we collected 5 larvae in 5 replicates per condition, rinsed them in PBS and placed them in a microtube with beads and $500 \mu \mathrm{L}$ PBS. Larvae were grinded using a Precellys 24 tissue homogenizer (Bertin Technologies, Montigny-le-Bretonneux, France. Settings: 6000 rpm, 2x30s, 30s pause). Optical

1125 Density at $0.629 \mathrm{~nm}$ was measured using a spectrophotometer SPECTROstarNano (BMG Labtech $\mathrm{GmbH}$, Ortenberg, Germany).

\section{RNA extraction of larval midgut}

1130 Larvae were reared as described for Developmental timing experiments and collected 1 day before the emergence of the first pupae. Larvae were dissected in PBS, and dissected anterior midguts were kept in RNAlater (Thermofisher, Waltham, Massachusetts, USA) before they were transferred to a microtube and flash-frozen. We used 10 guts for each replicate, and made 5 replicates for each condition. Samples 1135 were grinded using a Precellys 24 tissue homogenizer (Bertin Technologies, Montignyle-Bretonneux, France. Settings: 6500 rpm, 2x30s, 30s pause) and total RNA was 
extracted using a RNeasy kit (Macherey-Nagel, Hoerdt, France) following the instructions of the manufacturer.

\section{RNA extraction of Lp cultures}

Lp was grown in liquid imbalanced HD (Val-60\%) for five days (Consuegra et al., 2020a). RNA was extracted following a procedure adapted from (Nakashima et al., 2020). Briefly, cells were washed in PBS $1 \mathrm{X}$ and resuspended in $500 \mu \mathrm{L}$ TRI reagent

1145 (Qiagen, Hilden, Germany) with glass beads. Cells were vortexed for 3 minutes, incubated at $55^{\circ} \mathrm{C}$ for 30 minutes and centrifuged at $12000 \mathrm{~g}$ for 10 minutes at $4^{\circ} \mathrm{C}$. We added $100 \mu \mathrm{L}$ of chloroform, vortexed the tubes for three minutes and centrifuged at $12000 \mathrm{~g}$ for 10 minutes at $4^{\circ} \mathrm{C}$. We then collected the upper layer, added $160 \mu \mathrm{L}$ of absolute ethanol and applied it to a spin column of miRNeasy MiniKit (Qiagen, Hilden,

1150 Germany). RNA was purified using the miRNeasy MiniKit following the instructions of the manufacturer.

\section{RT-qPCR}

1155 We adjusted RNA concentrations and performed Reverse-Transcription (RT) on extracted RNAs using a SuperScript II RT kit (Thermofisher, Waltham, Massachusetts, USA) and random primers (Invitrogen, Waltham, Massachusetts, USA) following the instructions of the manufacturer. We then performed quantitative PCR using the primers GCN2-forward, GCN2-reverse, 4E-BP-forward, 4E-BP-reverse, rp49-forward,

1160 and rp49-reverse for Drosophila and 16S-forward, 16S-reverse, 23S-forward, 23Sreverse, gyrB-forward and gyrB-reverse for Lp (Table S2) using SYBR GreenER qPCR Supermix (Invitrogen, Waltham, Massachusetts, USA).

\section{RNA sequencing}

30 samples of total RNA isolated as previously described (5 replicates per condition) were used to build libraries using the SENSE mRNA-Seq Library Prep Kit V2 from Lexogen and following the RTS protocol. The libraries were single-indexed and pooled together in an equimolar manner in order to sequence 20 libraries at a time on a high-

1170 output run in a single-end mode (1x86bp) using a NextSeq500 Illumina sequencer. The two runs performed generated more than 535M reads each, resulting in an average of around 26-27 million reads per sample. Statistics of sequencing can be found in Table S3.

\section{Transcriptomic analyses}

We verified the quality of the samples using MultiQC on Galaxy (Afgan et al., 2016). Adapters and low-quality reads were removed with the help of Cutadapt on Galaxy (Martin, 2011). Clean reads were mapped using RNA STAR with default parameters 1180 (Dobin et al., 2013) against the genome of D. melanogaster (BDGP6.32.104). 
SARTools (Varet et al., 2016) was used for quality check of raw and normalized reads. Gene counts were normalized and tested for differential expression with DESEq2 package from R (Love et al., 2014). Genes were considered differentially expressed when the $\mathrm{BH}$ adjusted $\mathrm{p}$-value (P-adj) was lower than 0.05. Common significantly expressed genes in multiple conditions were tested for gene ontology (GO) enrichment with the clusterProfiler package from R (Yu et al., 2012) and non-redundant GO terms were reduced with the use of REVIGO (Supek et al., 2011).

\section{Figures and statistics}

Figures were created using the Prism GraphPad software, Biorender (BioRender.com) and Rstudio packages clusterProfiler (Yu et al., 2012), ggplot2 and plotly. Statistical analyses were performed using the Prism GraphPad software and RStudio.

\section{Supplementary tables}

\section{Table S1}

Differentially expressed genes and GO terms.

Table S2

Primers used in this study.

\section{Table S3}

Sequencing statistics.

\section{Acknowledgements}

We would like to thank Pr. Hyung Don Ryoo, Dr. Pierre Leopold, Dr. Nathalie Arquier, 1210 the Vienna Drosophila Resource Center and the Bloomington Stock Center for fly lines; Dr. David Duneau for his help with statistical analyses; Dr. Gilles Storelli for critical reading of the manuscript; Lucie Fallone and Meline Garcia, trainees of the Master Biosciences of ENS de Lyon for their help with experiments; the ArthroTools platform and the PLATIM platform of the SFR Biosciences (UAR3444/US8) for fly facility and

1215 microscopy equipments. Research in F. Leulier's lab is supported by the "Fondation pour la Recherche Médicale” («Equipe FRM DEQ20180339196) and the Scientific Breakthrough Project from Université de Lyon "Microbehave”. T. Grenier is funded by a PhD fellowship from ENS de Lyon. J. Consuegra is funded by a postdoctoral fellowship from the "Fondation pour la Recherche Médicale" (FRM, 1220 SPF20170938612).

\section{References}

Afgan, E., Baker, D., van den Beek, M., Blankenberg, D., Bouvier, D., Čech, M., Chilton, J., 1225 Clements, D., Coraor, N., Eberhard, C., et al. (2016). The Galaxy platform for accessible, 
reproducible and collaborative biomedical analyses: 2016 update. Nucleic Acids Res 44, W3W10.

Ahmed, S.M.H., Maldera, J.A., Krunic, D., Paiva-Silva, G.O., Pénalva, C., Teleman, A.A., and Edgar, B.A. (2020). Fitness trade-offs incurred by ovary-to-gut steroid signalling in Drosophila. Nature 584, 415-419.

Anthony, T.G., McDaniel, B.J., Byerley, R.L., McGrath, B.C., Cavener, D.R., McNurlan, M.A., and Wek, R.C. (2004). Preservation of liver protein synthesis during dietary leucine deprivation occurs at the expense of skeletal muscle mass in mice deleted for eIF2 kinase GCN2. J Biol Chem 279, 36553-36561.

1235 Armstrong, A.R., Laws, K.M., and Drummond-Barbosa, D. (2014). Adipocyte amino acid sensing controls adult germline stem cell number via the amino acid response pathway and independently of Target of Rapamycin signaling in Drosophila. Development 141, 44794488.

Axelsson, L., Rud, I., Naterstad, K., Blom, H., Renckens, B., Boekhorst, J., Kleerebezem, M., 1240 van Hijum, S., and Siezen, R.J. (2012). Genome sequence of the naturally plasmid-free Lactobacillus plantarum strain NC8 (CCUG 61730). J Bacteriol 194, 2391-2392.

Battley, E.H. (1988). Escherichia Coli and Salmonella Typhimurium. Cellular and Molecular Biology, Volume 1; Volume 2. Frederick C. Neidhardt, John L. Ingraham, Boris Magasanik , K. Brooks Low , Moselio Schaechter , H. Edwin Umbarger. The Quarterly Review of

1245 Biology 63, 463-464.

B'chir, W., Maurin, A.-C., Carraro, V., Averous, J., Jousse, C., Muranishi, Y., Parry, L., Stepien, G., Fafournoux, P., and Bruhat, A. (2013). The eIF2 $\alpha /$ ATF4 pathway is essential for stress-induced autophagy gene expression. Nucleic Acids Res 41, 7683-7699.

Berlanga, J.J., Ventoso, I., Harding, H.P., Deng, J., Ron, D., Sonenberg, N., Carrasco, L., and 1250 de Haro, C. (2006). Antiviral effect of the mammalian translation initiation factor 2alpha kinase GCN2 against RNA viruses. EMBO J 25, 1730-1740.

Bjordal, M., Arquier, N., Kniazeff, J., Pin, J.P., and Léopold, P. (2014). Sensing of Amino Acids in a Dopaminergic Circuitry Promotes Rejection of an Incomplete Diet in Drosophila. Cell 156, 510-521.

1255 Bonfini, A., Dobson, A.J., Duneau, D., Revah, J., Liu, X., Houtz, P., and Buchon, N. (2021). Multiscale analysis reveals that diet-dependent midgut plasticity emerges from alterations in both stem cell niche coupling and enterocyte size. ELife 10, e64125.

Broderick, N.A., Buchon, N., and Lemaitre, B. (2014). Microbiota-induced changes in drosophila melanogaster host gene expression and gut morphology. MBio 5, e01117-01114.

1260 Chakrabarti, S., Liehl, P., Buchon, N., and Lemaitre, B. (2012). Infection-Induced Host Translational Blockage Inhibits Immune Responses and Epithelial Renewal in the Drosophila Gut. Cell Host \& Microbe 12, 60-70.

Chen, K., Luan, X., Liu, Q., Wang, J., Chang, X., Snijders, A.M., Mao, J.-H., Secombe, J., Dan, Z., Chen, J.-H., et al. (2019). Drosophila Histone Demethylase KDM5 Regulates Social

1265 Behavior through Immune Control and Gut Microbiota Maintenance. Cell Host \& Microbe 25, 537-552.e8.

Colombani, J., Raisin, S., Pantalacci, S., Radimerski, T., Montagne, J., and Léopold, P. (2003). A Nutrient Sensor Mechanism Controls Drosophila Growth. Cell 114, 739-749. Combe, B.E., Defaye, A., Bozonnet, N., Puthier, D., Royet, J., and Leulier, F. (2014).

1270 Drosophila microbiota modulates host metabolic gene expression via IMD/NF- $\mathrm{B}$ signaling. PLoS One 9.

Consuegra, J., Grenier, T., Baa-Puyoulet, P., Rahioui, I., Akherraz, H., Gervais, H., Parisot, N., Silva, P. da, Charles, H., Calevro, F., et al. (2020a). Drosophila-associated bacteria differentially shape the nutritional requirements of their host during juvenile growth. PLOS

1275 Biology 18, e3000681. 
Consuegra, J., Grenier, T., Akherraz, H., Rahioui, I., Gervais, H., da Silva, P., and Leulier, F. (2020b). Metabolic Cooperation among Commensal Bacteria Supports Drosophila Juvenile Growth under Nutritional Stress. IScience 23, 101232.

Dang Do, A.N., Kimball, S.R., Cavener, D.R., and Jefferson, L.S. (2009). eIF2 $\alpha$ kinases GCN2 and PERK modulate transcription and translation of distinct sets of mRNAs in mouse liver. Physiol Genomics 38, 328-341.

Dever, T.E., Feng, L., Wek, R.C., Cigan, A.M., Donahue, T.F., and Hinnebusch, A.G. (1992). Phosphorylation of initiation factor $2 \alpha$ by protein kinase GCN2 mediates gene-specific translational control of GCN4 in yeast. Cell 68, 585-596.

1285 Dietzl, G., Chen, D., Schnorrer, F., Su, K.-C., Barinova, Y., Fellner, M., Gasser, B., Kinsey, K., Oppel, S., Scheiblauer, S., et al. (2007). A genome-wide transgenic RNAi library for conditional gene inactivation in Drosophila. Nature 448, 151-156.

Dobin, A., Davis, C.A., Schlesinger, F., Drenkow, J., Zaleski, C., Jha, S., Batut, P., Chaisson, M., and Gingeras, T.R. (2013). STAR: ultrafast universal RNA-seq aligner. Bioinformatics

$1290 \quad 29,15-21$.

Domínguez Rubio, A.P., Martínez, J.H., Martínez Casillas, D.C., Coluccio Leskow, F., Piuri, M., and Pérez, O.E. (2017). Lactobacillus casei BL23 Produces Microvesicles Carrying Proteins That Have Been Associated with Its Probiotic Effect. Front Microbiol 8.

Dong, J., Qiu, H., Garcia-Barrio, M., Anderson, J., and Hinnebusch, A.G. (2000). Uncharged

1295 tRNA Activates GCN2 by Displacing the Protein Kinase Moiety from a Bipartite tRNABinding Domain. Molecular Cell 6, 269-279.

Donnelly, N., Gorman, A.M., Gupta, S., and Samali, A. (2013). The eIF2 $\alpha$ kinases: their structures and functions. Cell Mol Life Sci 70, 3493-3511.

Elgart, M., Stern, S., Salton, O., Gnainsky, Y., Heifetz, Y., and Soen, Y. (2016). Impact of gut 1300 microbiota on the fly's germ line. Nat Commun 7, 11280.

Erkosar, B., Storelli, G., Defaye, A., and Leulier, F. (2013). Host-intestinal microbiota mutualism: "learning on the fly." Cell Host \& Microbe 13, 8-14.

Erkosar, B., Storelli, G., Mitchell, M., Bozonnet, L., Bozonnet, N., and Leulier, F. (2015). Pathogen virulence impedes mutualist-mediated enhancement of host juvenile growth via

1305 inhibition of protein digestion. Cell Host Microbe 18, 445-455.

Gallinetti, J., Harputlugil, E., and Mitchell, J.R. (2013). Amino acid sensing in dietaryrestriction-mediated longevity: roles of signal-transducing kinases GCN2 and TOR. Biochem J 449, 1-10.

Gnainsky, Y., Zfanya, N., Elgart, M., Omri, E., Brandis, A., Mehlman, T., Itkin, M.,

1310 Malitsky, S., Adamski, J., and Soen, Y. (2021). Systemic Regulation of Host Energy and Oogenesis by Microbiome-Derived Mitochondrial Coenzymes. Cell Reports 34, 108583. Goberdhan, D.C.I., Wilson, C., and Harris, A.L. (2016). Amino Acid Sensing by mTORC1: Intracellular Transporters Mark the Spot. Cell Metab 23, 580-589.

Gould, A.L., Zhang, V., Lamberti, L., Jones, E.W., Obadia, B., Korasidis, N., Gavryushkin,

1315 A., Carlson, J.M., Beerenwinkel, N., and Ludington, W.B. (2018). Microbiome interactions shape host fitness. Proc. Natl. Acad. Sci. U.S.A. 115, E11951-E11960.

Gourse, R.L., Takebe, Y., Sharrock, R.A., and Nomura, M. (1985). Feedback regulation of rRNA and tRNA synthesis and accumulation of free ribosomes after conditional expression of rRNA genes. Proc Natl Acad Sci U S A 82, 1069-1073.

1320 Grallert, B., and Boye, E. (2007). The Gcn2 Kinase as a Cell Cycle Regulator. Cell Cycle 6, 2768-2772.

Guo, F., and Cavener, D.R. (2007). The GCN2 eIF2 $\alpha$ Kinase Regulates Fatty-Acid Homeostasis in the Liver during Deprivation of an Essential Amino Acid. Cell Metabolism 5, 103-114.

1325 Harding, H.P., Zhang, Y., Zeng, H., Novoa, I., Lu, P.D., Calfon, M., Sadri, N., Yun, C., 
1330 Hothorn, T., Bretz, F., and Westfall, P. (2008). Simultaneous inference in general parametric models. Biom J 50, 346-363.

Iatsenko, I., Boquete, J.-P., and Lemaitre, B. (2018). Microbiota-derived lactate activates production of Reactive Oxygen Species by the intestinal NADPH oxidase Nox and shortens Drosophila Lifespan. Immunity 49, 929-942.e5.

1335 Jugder, B.-E., Kamareddine, L., and Watnick, P.I. (2021). Microbiota-derived acetate activates intestinal innate immunity via the Tip60 histone acetyltransferase complex. Immunity S1074-7613(21)00223-5.

Kamareddine, L., Robins, W.P., Berkey, C.D., Mekalanos, J.J., and Watnick, P.I. (2018). The Drosophila Immune Deficiency Pathway Modulates Enteroendocrine Function and Host

1340 Metabolism. Cell Metabolism.

Kang, M.-J., Vasudevan, D., Kang, K., Kim, K., Park, J.-E., Zhang, N., Zeng, X., Neubert, T.A., Marr, M.T., and Ryoo, H.D. (2017). 4E-BP is a target of the GCN2-ATF4 pathway during Drosophila development and aging. J Cell Biol 216, 115-129.

Keebaugh, E.S., Yamada, R., Obadia, B., Ludington, W.B., and Ja, W.W. (2018). Microbial

1345 quantity impacts Drosophila nutrition, development, and lifespan. IScience 4, 247-259.

Keebaugh, E.S., Yamada, R., and Ja, W.W. (2019). The Nutritional Environment Influences the Impact of Microbes on Drosophila melanogaster Life Span. MBio 10.

Kilstrup, M., Hammer, K., Ruhdal Jensen, P., and Martinussen, J. (2005). Nucleotide metabolism and its control in lactic acid bacteria. FEMS Microbiology Reviews 29, 555-590.

1350 Kim, B., Kanai, M.I., Oh, Y., Kyung, M., Kim, E.-K., Jang, I.-H., Lee, J.-H., Kim, S.-G., Suh, G.S.B., and Lee, W.-J. (2021). Response of the microbiome-gut-brain axis in Drosophila to amino acid deficit. Nature 1-5.

Kim, K., Park, J.-E., Yeom, J., Park, N., Trần, T.-X.T., and Kang, M.-J. (2020). Tissuespecific roles of GCN2 in aging and autosomal dominant retinitis pigmentosa. Biochem

1355 Biophys Res Commun 533, 1054-1060.

Koeppen, K., Hampton, T.H., Jarek, M., Scharfe, M., Gerber, S.A., Mielcarz, D.W., Demers, E.G., Dolben, E.L., Hammond, J.H., Hogan, D.A., et al. (2016). A Novel Mechanism of HostPathogen Interaction through sRNA in Bacterial Outer Membrane Vesicles. PLOS Pathogens 12, e1005672.

1360 Krishnamoorthy, J., Mounir, Z., Raven, J., and Koromilas, A. (2008). The eIF2 $\alpha$ kinases inhibit vesicular stomatitis virus replication independently of eIF2 phosphorylation. Cell Cycle 7, 2346-2351.

Kuhn, A., Koch, H.-G., and Dalbey, R.E. (2017). Targeting and Insertion of Membrane Proteins. EcoSal Plus 7.

1365 Laeger, T., Albarado, D.C., Burke, S.J., Trosclair, L., Hedgepeth, J.W., Berthoud, H.-R., Gettys, T.W., Collier, J.J., Münzberg, H., and Morrison, C.D. (2016). Metabolic Responses to Dietary Protein Restriction Require an Increase in FGF21 that Is Delayed by the Absence of GCN2. Cell Reports 16, 707-716.

Laplante, M., and Sabatini, D.M. (2009). mTOR signaling at a glance. J Cell Sci 122, 358913703594.

Lee, Y.S., Shibata, Y., Malhotra, A., and Dutta, A. (2009). A novel class of small RNAs: tRNA-derived RNA fragments (tRFs). Genes Dev 23, 2639-2649.

Leitão-Gonçalves, R., Carvalho-Santos, Z., Francisco, A.P., Fioreze, G.T., Anjos, M., Baltazar, C., Elias, A.P., Itskov, P.M., Piper, M.D.W., and Ribeiro, C. (2017). Commensal

1375 bacteria and essential amino acids control food choice behavior and reproduction. PLOS 
Biology 15, e2000862.

Lesperance, D.N., and Broderick, N.A. (2020). Microbiomes as modulators of Drosophila melanogaster homeostasis and disease. Current Opinion in Insect Science 39, 84-90. Li, M., Lee, K., Hsu, M., Nau, G., Mylonakis, E., and Ramratnam, B. (2017). Lactobacillusderived extracellular vesicles enhance host immune responses against vancomycin-resistant enterococci. BMC Microbiol 17.

Love, M.I., Huber, W., and Anders, S. (2014). Moderated estimation of fold change and dispersion for RNA-seq data with DESeq2. Genome Biology 15, 550.

Lunt, S.Y., and Vander Heiden, M.G. (2011). Aerobic Glycolysis: Meeting the Metabolic

1385 Requirements of Cell Proliferation. Annual Review of Cell and Developmental Biology 27, 441-464.

Ma, X.M., and Blenis, J. (2009). Molecular mechanisms of mTOR-mediated translational control. Nat Rev Mol Cell Biol 10, 307-318.

Maguin, E., Prévost, H., Ehrlich, S.D., and Gruss, A. (1996). Efficient insertional mutagenesis

1390 in lactococci and other gram-positive bacteria. J Bacteriol 178, 931-935.

Malzer, E., Szajewska-Skuta, M., Dalton, L.E., Thomas, S.E., Hu, N., Skaer, H., Lomas, D.A., Crowther, D.C., and Marciniak, S.J. (2013). Coordinate regulation of eIF2 $\alpha$ phosphorylation by PPP1R15 and GCN2 is required during Drosophila development. J Cell Sci 126, 1406-1415.

1395 Malzer, E., Dominicus, C.S., Chambers, J.E., Dickens, J.A., Mookerjee, S., and Marciniak, S.J. (2018). The integrated stress response regulates BMP signalling through effects on translation. BMC Biol 16.

Martin, M. (2011). Cutadapt removes adapter sequences from high-throughput sequencing reads. EMBnet.Journal 17, 10-12.

1400 Martino, M.E., Bayjanov, J.R., Caffrey, B.E., Wels, M., Joncour, P., Hughes, S., Gillet, B., Kleerebezem, M., van Hijum, S.A.F.T., and Leulier, F. (2016). Nomadic lifestyle of Lactobacillus plantarum revealed by comparative genomics of 54 strains isolated from different habitats. Environ. Microbiol. 18, 4974-4989.

Masson, G.R. (2019). Towards a model of GCN2 activation. Biochem Soc Trans 47, 1481-

14051488.

Matos, R.C., Schwarzer, M., Gervais, H., Courtin, P., Joncour, P., Gillet, B., Ma, D., Bulteau, A.-L., Martino, M.E., Hughes, S., et al. (2017). D-Alanylation of teichoic acids contributes to Lactobacillus plantarum-mediated Drosophila growth during chronic undernutrition. Nat Microbiol 2,1635-1647.

1410 Moriano-Gutierrez, S., Bongrand, C., Essock-Burns, T., Wu, L., McFall-Ngai, M.J., and Ruby, E.G. (2020). The noncoding small RNA SsrA is released by Vibrio fischeri and modulates critical host responses. PLOS Biology 18, e3000934.

Nakashima, Y., Shiiyama, N., Urabe, T., Yamashita, H., Yasuda, S., Igoshi, K., and Kinoshita, H. (2020). Functions of small RNAs in Lactobacillus casei-Pediococcus group of

1415 lactic acid bacteria using fragment analysis. FEMS Microbiology Letters 367.

Newell, P.D., and Douglas, A.E. (2014). Interspecies interactions determine the impact of the gut microbiota on nutrient allocation in Drosophila melanogaster. Appl. Environ. Microbiol. 80, 788-796.

Olsen, D.S., Jordan, B., Chen, D., Wek, R.C., and Cavener, D.R. (1998). Isolation of the gene 1420 encoding the Drosophila melanogaster homolog of the Saccharomyces cerevisiae GCN2 eIF2alpha kinase. Genetics 149, 1495-1509.

Overend, G., Luo, Y., Henderson, L., Douglas, A.E., Davies, S.A., and Dow, J.A.T. (2016).

Molecular mechanism and functional significance of acid generation in the Drosophila midgut. Sci Rep 6, 27242.

1425 Palmer, B.R., and Marinus, M.G. (1994). The dam and dcm strains of Escherichia coli--a 
review. Gene 143, 1-12.

Piper, M.D. (2017). Using artificial diets to understand the nutritional physiology of Drosophila melanogaster. Curr Opin Insect Sci 23, 104-111.

Piper, M.D.W., Soultoukis, G.A., Blanc, E., Mesaros, A., Herbert, S.L., Juricic, P., He, X., Atanassov, I., Salmonowicz, H., Yang, M., et al. (2017). Matching dietary amino acid balance to the in silico-translated exome optimizes growth and reproduction without cost to lifespan. Cell Metabolism 25, 610-621.

Redhai, S., Pilgrim, C., Gaspar, P., Giesen, L. van, Lopes, T., Riabinina, O., Grenier, T., Milona, A., Chanana, B., Swadling, J.B., et al. (2020). An intestinal zinc sensor regulates

1435 food intake and developmental growth. Nature 580, 263-268.

Reiff, T., Jacobson, J., Cognigni, P., Antonello, Z., Ballesta, E., Tan, K.J., Yew, J.Y., Dominguez, M., and Miguel-Aliaga, I. (2015). Endocrine remodelling of the adult intestine sustains reproduction in Drosophila. ELife 4, e06930.

Ren, B., Wang, X., Duan, J., and Ma, J. (2019). Rhizobial tRNA-derived small RNAs are

1440 signal molecules regulating plant nodulation. Science 365, 919-922.

Rodrigues, Y.K., Bergen, E. van, Alves, F., Duneau, D., and Beldade, P. (2021). Additive and non-additive effects of day and night temperatures on thermally plastic traits in a model for adaptive seasonal plasticity. Evolution 75, 1805-1819.

Ryu, J.-H., Kim, S.-H., Lee, H.-Y., Bai, J.Y., Nam, Y.-D., Bae, J.-W., Lee, D.G., Shin, S.C.,

1445 Ha, E.-M., and Lee, W.-J. (2008). Innate immune homeostasis by the homeobox gene Caudal and commensal-gut mutualism in Drosophila. Science 319, 777-782.

Saguir, F.M., and de Nadra, M.C.M. (2007). Improvement of a chemically defined medium for the sustained growth of Lactobacillus plantarum: nutritional requirements. Curr.

Microbiol. 54, 414-418.

1450 Schindelin, J., Arganda-Carreras, I., Frise, E., Kaynig, V., Longair, M., Pietzsch, T., Preibisch, S., Rueden, C., Saalfeld, S., Schmid, B., et al. (2012). Fiji: an open-source platform for biological-image analysis. Nat Methods 9, 676-682.

Selosse, M.-A., Bessis, A., and Pozo, M.J. (2014). Microbial priming of plant and animal immunity: symbionts as developmental signals. Trends in Microbiology 22, 607-613.

1455 Sharon, G., Segal, D., Ringo, J.M., Hefetz, A., Zilber-Rosenberg, I., and Rosenberg, E. (2010). Commensal bacteria play a role in mating preference of Drosophila melanogaster. PNAS 107, 20051-20056.

Shin, S.C., Kim, S.-H., You, H., Kim, B., Kim, A.C., Lee, K.-A., Yoon, J.-H., Ryu, J.-H., and Lee, W.-J. (2011). Drosophila microbiome modulates host developmental and metabolic

1460 homeostasis via insulin signaling. Science 334, 670-674.

Staubach, F., Baines, J.F., Künzel, S., Bik, E.M., and Petrov, D.A. (2013). Host Species and Environmental Effects on Bacterial Communities Associated with Drosophila in the Laboratory and in the Natural Environment. PLOS ONE 8, e70749.

Storelli, G., Defaye, A., Erkosar, B., Hols, P., Royet, J., and Leulier, F. (2011). Lactobacillus

1465 plantarum promotes Drosophila systemic growth by modulating hormonal signals through TOR-dependent nutrient sensing. Cell Metab. 14, 403-414.

Storelli, G., Strigini, M., Grenier, T., Bozonnet, L., Schwarzer, M., Daniel, C., Matos, R., and Leulier, F. (2018). Drosophila perpetuates nutritional mutualism by promoting the fitness of its intestinal symbiont Lactobacillus plantarum. Cell Metab 27, 362-377.e8.

1470 Supek, F., Bošnjak, M., Škunca, N., and Šmuc, T. (2011). REVIGO Summarizes and Visualizes Long Lists of Gene Ontology Terms. PLOS ONE 6, e21800.

Tattoli, I., Sorbara, M.T., Vuckovic, D., Ling, A., Soares, F., Carneiro, L.A.M., Yang, C., Emili, A., Philpott, D.J., and Girardin, S.E. (2012). Amino Acid Starvation Induced by Invasive Bacterial Pathogens Triggers an Innate Host Defense Program. Cell Host \& Microbe $147511,563-575$. 
Tennessen, J.M., and Thummel, C.S. (2011). Coordinating growth and review maturation insights from Drosophila. Curr Biol 21, R750-R757.

Teske, B.F., Baird, T.D., and Wek, R.C. (2011). Chapter Nineteen - Methods for Analyzing eIF2 Kinases and Translational Control in the Unfolded Protein Response. In Methods in Enzymology, P.M. Conn, ed. (Academic Press), pp. 333-356.

Teusink, B., van Enckevort, F.H.J., Francke, C., Wiersma, A., Wegkamp, A., Smid, E.J., and Siezen, R.J. (2005). In silico reconstruction of the metabolic pathways of Lactobacillus plantarum: comparing predictions of nutrient requirements with those from growth experiments. Appl Environ Microbiol 71, 7253-7262.

1485 Therneau, T.M., and Grambsch, P.M. (2000). Modeling Survival Data: Extending the Cox Model (New York: Springer-Verlag).

Vandehoef, C., Molaei, M., and Karpac, J. (2020). Dietary Adaptation of Microbiota in Drosophila Requires NF-кB-Dependent Control of the Translational Regulator 4E-BP. Cell Rep 31, 107736.

1490 Varet, H., Brillet-Guéguen, L., Coppée, J.-Y., and Dillies, M.-A. (2016). SARTools: A DESeq2- and EdgeR-Based R Pipeline for Comprehensive Differential Analysis of RNA-Seq Data. PLOS ONE 11, e0157022.

Vasudevan, D., Clark, N.K., Sam, J., Cotham, V.C., Ueberheide, B., Marr, M.T., and Ryoo, H.D. (2017). The GCN2-ATF4 signaling pathway induces 4E-BP to bias translation and boost 1495 antimicrobial peptide synthesis in response to bacterial infection. Cell Reports 21, 20392047.

Warburg, O. (1956). On the Origin of Cancer Cells. Science 123, 309-314.

Wek, R.C., and Cavener, D.R. (2007). Translational Control and the Unfolded Protein Response. Antioxidants \& Redox Signaling 9, 2357-2372.

1500 Wek, S.A., Zhu, S., and Wek, R.C. (1995). The histidyl-tRNA synthetase-related sequence in the eIF-2 alpha protein kinase GCN2 interacts with tRNA and is required for activation in response to starvation for different amino acids. Mol Cell Biol 15, 4497-4506.

West, C.L., Stanisz, A.M., Mao, Y.-K., Champagne-Jorgensen, K., Bienenstock, J., and Kunze, W.A. (2020). Microvesicles from Lactobacillus reuteri (DSM-17938) completely

1505 reproduce modulation of gut motility by bacteria in mice. PLOS ONE 15, e0225481.

Wong, S.Y., Javid, B., Addepalli, B., Piszczek, G., Strader, M.B., Limbach, P.A., and Barry, C.E. (2013). Functional role of methylation of G518 of the 16S rRNA 530 loop by GidB in Mycobacterium tuberculosis. Antimicrob. Agents Chemother. 57, 6311-6318.

Yamada, R., Deshpande, S.A., Bruce, K.D., Mak, E.M., and Ja, W.W. (2015). Microbes

1510 promote amino acid harvest to rescue undernutrition in Drosophila. Cell Rep. Yu, G., Wang, L.-G., Han, Y., and He, Q.-Y. (2012). clusterProfiler: an R Package for Comparing Biological Themes Among Gene Clusters. OMICS 16, 284-287.

Zhang, P., McGrath, B.C., Reinert, J., Olsen, D.S., Lei, L., Gill, S., Wek, S.A., Vattem, K.M., Wek, R.C., Kimball, S.R., et al. (2002). The GCN2 eIF2 $\alpha$ Kinase Is Required for Adaptation

1515 to Amino Acid Deprivation in Mice. Mol Cell Biol 22, 6681-6688.

Zhu, S., and Wek, R.C. (1998). Ribosome-binding Domain of Eukaryotic Initiation Factor-2 Kinase GCN2 Facilitates Translation Control *. Journal of Biological Chemistry 273, 18081814. 